\title{
Inelastic and quasi-elastic neutron scattering. Application to soft-matter
}

\author{
Quentin Berrod ${ }^{1}$, Karine Lagrené ${ }^{1}$, Jacques Ollivier ${ }^{2}$, and Jean-Marc Zanotti ${ }^{1}$ „ \\ ${ }^{1}$ Laboratoire Léon Brillouin (CEA-CNRS), Université Paris-Saclay, \\ CEA Saclay, 91191 Gif-sur-Yvette Cedex, France \\ ${ }^{2}$ Institut Laue-Langevin, 38042 Grenoble Cedex, France
}

\begin{abstract}
Microscopic dynamical events control many of the physical processes at play in condensed matter: transport, magnetism, catalysis and even function of biological assemblies. Inelastic (INS) and Quasi-Elastic Neutron Scattering (QENS) are irreplaceable probes of these phenomena. These experimental techniques reveal the displacements of atoms and molecules over distances spanning from angstroms to a few tens of nanometers, on time scales ranging from a fraction of picoseconds to microseconds. In this context, the different INS and QENS machines (Time-of-Flight (ToF), Backscattering (BS) and Neutron spin-echo (NSE)) stand at a central position. After introducing an underlying basic theoretical toolbox for neutron scattering, the principles and key elements of a ToF measurement are described. While, here, we mainly focus on disk choppers spectrometers, all the INS/QENS instruments share a common ground: they directly and simultaneously probe correlation functions in both time and space, so that the scattering vector $(Q)$ dependence of the systems characteristic time(s) can be measured. To illustrate, the potentialities of the technique in the field of soft-matter, we show a multiscale approach of the dynamics of a polymer melt. The system is probed from the molecular to the mesoscopic scale ( 1 ps to $0.6 \mu$ s and 0.1 to $40 \mathrm{~nm}$ ), in bulk and under nanometric confinement. We address the different dynamical modes of a high mass entangled polymer chain: local monomer dynamics, Rouse modes up to the reptation process. This study exemplifies that, used in conjunction with hydrogen/deuterium isotopic effects, high resolution QENS can be bridged to the Zero Average Contrast (ZAC) method to probe, in a non destructive way, the dynamics of a single polymer chain in bulk but also under severe nanometric confinement. Connection and complementarity of the neutron derived analysis with Pulsed-Field Gradient and Relaxation NMR techniques are discussed.
\end{abstract}

\footnotetext{
^e-mail: jmzanotti@cea.fr
} 


\section{Contents}

1 Introduction $\quad \mathbf{3}$

1.1 Phenomena accessible by neutron scattering. . . . . . . . . . . . . 3

1.2 Spatial and temporal ranges probed by neutron time-of-flight. . . . . . . . . . . . 4

2 Neutron scattering: Few theoretical elements 5

2.1 Basic principles. . . . . . . . . . . . . . . . . 5

2.2 Coherent and incoherent scattering. . . . . . . . . . . . . 6

2.3 The van Hove formalism. . . . . . . . . . . . . . . . . . . . . . 6

2.4 Taking advantage of isotopic effects. . . . . . . . . . . . . 8

2.5 Derivation of $S_{i n c}(Q, \omega)$ for selected examples of dynamical modes. . . . . . . . . 8

2.5.1 Long range translational and jump diffusion. . . . . . . . . . . . 8

2.5.2 Diffusion in restricted geometry. The notion of EISF. . . . . . . . . . . . 10

2.5 .3 Vibrational dynamics. . . . . . . . . . . . . . . . . . . . 13

2.5.4 The total dynamical structure factor. . . . . . . . . . . . . . . . . . . . . . 14

2.6 Aspects related to the sample temperature . . . . . . . . . . . . . . . 15

3 Instrumentation $\quad 16$

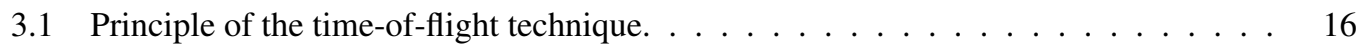

3.2 Implementation. . . . . . . . . . . . . . . . . . . . . 17

3.2.1 Monochromatisation. . . . . . . . . . . . . . . 18

3.2.2 Direct geometry time-of-flight instruments: The chopper spectrometers. . . . 20

3.2.3 Inverted geometry time-of-flight instrument. . . . . . . . . . . . . . 20

3.3 Experimental energy resolution effects and consequences. . . . . . . . . . . . 21

4 The tools at work: A multiscale study of a polymer melt in bulk and under nanometric confinement. Connection to NMR

4.1 Polymer dynamics in a nutshell. . . . . . . . . . . . . . . . 23

4.2 Local polymer dynamics: A neutron-derived physical model. . . . . . . . . . . . . 25

4.2.1 QENS derived dynamical susceptibility as a tool to determine the actual number of dynamical contributions. . . . . . . . . . . . . . 25

4.2.2 A physical appealing model of the local polymer dynamics: The building blocks.

4Q.3 Bridging the QENS and the NMR relaxation models. . . . . . . . . . . . . . 29

4.3 The Rouse regime. . . . . . . . . . . . . . . . . . . . . . 30

4.4 The reptation regime: Observing a single polymer chain (Zero Average Contrast). . . 32

4.5 Multiscale study of a polymer melt: Conclusion. . . . . . . . . . . . . . . 34 


\section{Introduction}

Within the framework of the wave-particle duality, a wave can be associated to a neutron. The thermal neutron beams ${ }^{1}$ made available to the scientific community are designed to provide a wavelength range of the order of magnitude of interatomic distances. The interaction of a neutron beam with matter thus leads to a scattering phenomenon, and its analysis gives access to the local structure of the sample. A second particularly interesting feature of thermal neutrons is that their energy is of the order of magnitude of many excitations at play in condensed matter. The analysis of the energy exchanged between a neutron and the sample thus also makes it possible to access the dynamics of the system. A key point to keep in mind is that the underlying physical mechanism leading to the scattering phenomenon is a nuclear process driven by a spin-spin interaction between the spin of the incident neutron and the nuclear spin of the target nucleus. Neutron scattering then offers the interesting feature to play with strong isotopic effects. In the field of soft-matter, the Hydrogen/Deuterium (H/D) isotopic effect can be advantageously considered to $i$ ) mask a dynamical contribution of the system (solvent, side-chains...) and $i$ ) finely balance the incoherent and coherent scattering to assess individual (selfdiffusion coefficient for example) or collective (phonons) dynamical information. Also, the scattering vector $(\vec{Q})$ dependence can be used to access the geometry of the atomic motions (jump distances in a jump-diffusion process for example). The first sections of the manuscript are devoted to provide more detailed information of the points/properties described above. For the sake of consistency, throughout this manuscript, all the specific example, illustrating this lecture will be related to neutron experiments on a single polymer system: Poly(Etylene Oxide) $\left(\mathrm{PEO}^{2},-\left[\mathrm{CH}_{2}-\mathrm{CH}_{2}-\mathrm{O}\right]_{n}-, 35 \mathrm{~kg} \cdot \mathrm{mol}^{-1}\right.$ i.e. above the critical entanglement mass $\left.M_{e}=3.5 \mathrm{~kg} \cdot \mathrm{mol}^{-1}\right)$.

\subsection{Phenomena accessible by neutron scattering}

The time-of-flight (ToF) technique allows the study of dynamical phenomena of nuclear or magnetic origin. The quasi-elastic region, which extends up to a few meV around the elastic peak (zero energy transfer point: $\hbar \omega=0 \mathrm{meV}$ ) is characteristic of phenomena and/or relaxations processes in the system. Over the whole energy range, from 0 to several hundreds $\mathrm{meV}$, the signal can also corresponds to the exchange of quanta of energy between the neutron and the sample (Fig.1). The energy transfer range above $1 \mathrm{meV}$ is often referred to as spectroscopic (neutron spectroscopy).

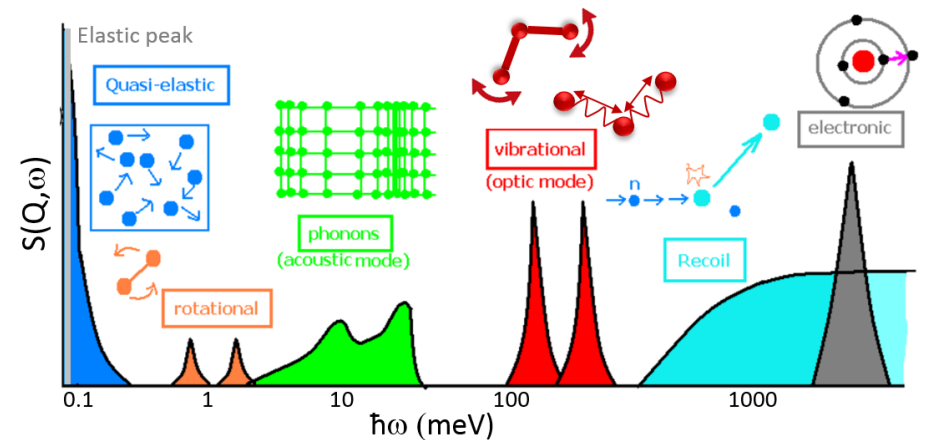

Figure 1. Schematics of the dynamical structure factors, $S(Q, \omega)$, of a selection of phenomena accessible by quasi-elastic and inelastic neutron scattering (from [1]).

\footnotetext{
${ }^{1}$ Thermal neutrons: neutrons of $1.8 \AA$ wavelength. The associated energy is in the order of magnitude of the thermal agitation at room temperature i.e. $293 \mathrm{~K}, 25.25 \mathrm{meV}, 203.65 \mathrm{~cm}^{-1}, 2200 \mathrm{~m} . \mathrm{s}^{-1}$ or $6.11 \mathrm{Thz}$.

${ }^{2}$ Fully hydrogenated and fully deuterated PEO will be respectively designed by ${ }^{h} \mathrm{PEO}$ and ${ }^{d} \mathrm{PEO}$.
} 


\subsection{Spatial and temporal ranges probed by neutron time-of-flight}

The Fig.2 specifies the field of action of time-of-flight spectrometers in the neutron techniques instrumental scenery. They allow to cover the wide range of the $(Q, \omega)$ domain, intermediate between the filters instruments (triple axis) and the high resolution instruments like the backscattering or spin-echo spectrometers. This representation, however, disregards the counting times required for measuring an exploitable signal and certain technical limitations: the sample quantity and/or the low scattering may, for example, be an obstacle to backscattering studies. In spin-echo, the absence of polarization - induced by a coherent/incoherent scattering competition - can make it impossible to measure the dynamics of the system in certain domains of scattering vector.

The temporal range probed by time-of-flight calls upon a fundamental instrumental notion which

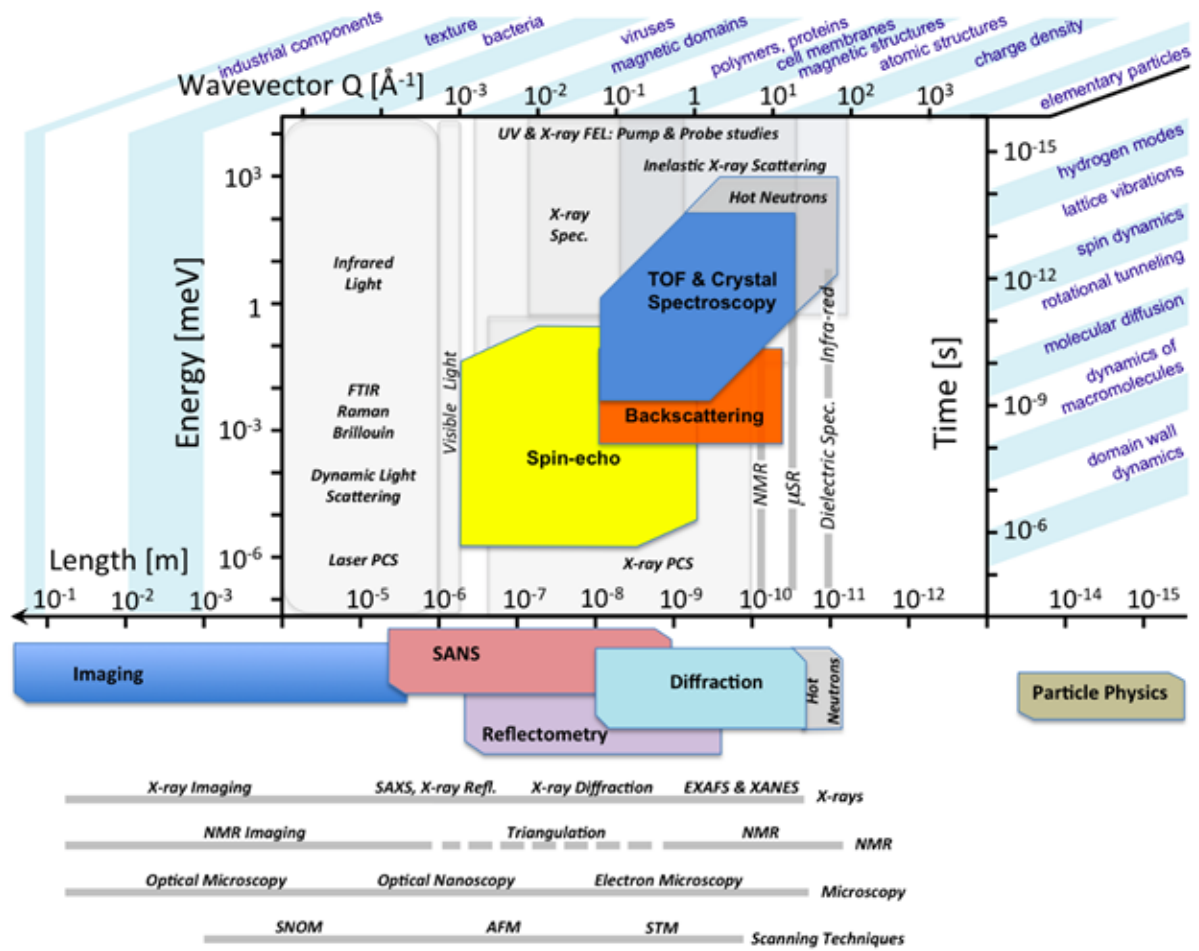

Figure 2. a) Complementarity of inelastic neutron scattering (triple axis also called "filter techniques"), timeof-flight, backscattering and neutron spin-echo) with other spectroscopic techniques. The spatial $(r[\AA] \approx$ $\left.2 \pi / Q\left[\AA^{-1}\right]\right)$ and temporal $(t[p s] \approx 0.658 / \hbar \omega[\mathrm{meV}])$ regions associated with the main physical phenomena potentially observable are shown. Connection, not shown on this graph, to thermodynamic quantities, like the specific-heat is also possible (see Eq.25). One can note that neutron scattering has the capability to simultaneously probe a significant region of this spatial and temporal map. Also, the spatial dependence of the correlation time is directly accessible. From the European Spallation Source Web site (https://europeanspallationsource.se/scienceusing-neutrons).

will be discussed in greater detail hereafter: the energy resolution. It results from instrumental imperfections. This energy resolution limits not only the precision of the measurement but also defines the upper limit to the (longest) accessible correlation times (see section 3.3). 


\section{Neutron scattering: Few theoretical elements}

\subsection{Basic principles}

As in all radiation scattering experiments, a neutron scattering experiment consists of illuminating a sample with a neutron beam of wave vector $\overrightarrow{k_{0}}$, wavelength $\lambda_{0}$ and energy $E_{0}$. Under the effect of the interaction $V(\vec{r}, t)$ between the neutron and the system, downstream of the sample, the radiation is characterized by a wave vector $\overrightarrow{k_{1}}$ and an energy $E_{1}$ (Fig.3). The diffusion angle, $2 \theta$, is defined as the angle between the vectors $\overrightarrow{k_{1}}$ and $\overrightarrow{k_{0}}$. The energy of a neutron with wavevector $\vec{k}$ is:

$$
E=\hbar \omega=\frac{\hbar^{2}|\vec{k}|^{2}}{2 m_{n}}, k=\frac{2 \pi}{\lambda}
$$

where $m_{n}$ is the neutron mass and $\hbar$, the Planck constant divided by $2 \pi$. The momentum and energy conservation rules lead to:

$$
\begin{aligned}
\vec{Q} & =\overrightarrow{k_{1}}-\overrightarrow{k_{0}} \\
\hbar \omega & =\hbar\left(\omega_{0}-\omega_{1}\right)
\end{aligned}
$$

where $\vec{Q}^{3}$ is the scattering vector and $\hbar \omega$ is the energy gained or lost by the neutron. Below, by convention, the negative values of $\omega$ will correspond to an energy loss of the sample, therefore an energy gain of the neutron. Similar to a classical collision (e.g. like at the pool game), the conservation law of momentum and energy imposes the kinematic condition:

$$
\frac{Q^{2}}{k_{0}^{2}}=2-\frac{\omega}{\omega_{0}}-2 \cos (2 \theta) \sqrt{1-\frac{\omega}{\omega_{0}}}
$$

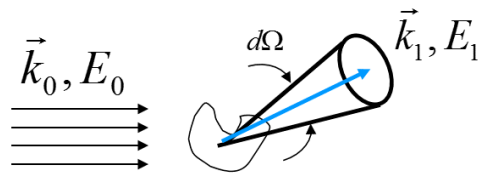

$$
\begin{aligned}
& \vec{Q}=\vec{k}_{1}-\vec{k}_{0} \\
& \hbar \omega=E_{i}-E_{f}
\end{aligned}
$$

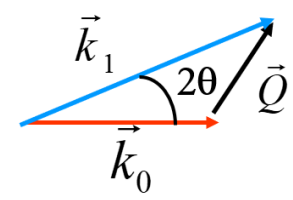

Figure 3. Principle of a neutron scattering experiment. As a result of interaction with the sample, incident neutrons $\left(\overrightarrow{k_{0}}\right.$ and $\left.\omega_{0}\right)$ can undergo a wave vector and energy changes: respectively $\vec{Q}=\overrightarrow{k_{1}}-\overrightarrow{k_{0}}$ and $\hbar \omega=\hbar\left(\omega_{0}-\omega_{1}\right)$. In this general case of an inelastic interaction $(\hbar \omega \neq 0), Q$ is a function of $\theta$ and $\omega$ (Eq.3). For an elastic scattering process $\overrightarrow{k_{1}}=\overrightarrow{k_{0}}\left(\right.$ i.e. $\omega_{0}=\omega_{1}$ ), the elastic scattering vector is $\vec{Q}_{e l}=\frac{4 \pi}{\lambda} \sin (\theta) \cdot \vec{u}$, where $\vec{u}$ denotes a unit vector (not shown). In the reciprocal domain, i.e. the domain where the neutron detector directly operates, the two working quantities are $Q$ and $\omega$. The two dual quantities in the real space, i.e. the domain of the sample, are the time, $t$, and space, $r$. One can switch from one domain to the other by Fourier Transform over $r$ and $t$ (Eq.4).

Experimentally, one measures [2] the differential scattering cross-section i.e. the number of neutrons scattered over a solid-angle $d \Omega$ and by energy transfer $d \omega$. This quantity is ruled by the time dependence of the positions $\overrightarrow{r_{i}}(t)$ of the atoms of the system. ${ }^{4}$

$$
\frac{d^{2} \sigma}{d \Omega d \omega}=\frac{1}{2 \pi} \frac{k_{1}}{k_{0}} \sum_{i, j}\left\langle b_{i} b_{j}\right\rangle \int_{-\infty}^{+\infty}\left\langle e^{i \vec{Q} \vec{r}_{i}(0)} e^{-i \vec{Q} \vec{Q}_{j}(t)}\right\rangle e^{-i \omega t} \cdot d t
$$

\footnotetext{
${ }^{3}$ From now on, the scattering vector $\vec{Q}$ will be simply written $Q$.

${ }^{4}$ From now on, the time dependence of the position $\vec{r}_{i}(t)$ will be written $r_{i}(t)$
} 


\subsection{Coherent and incoherent scattering}

The neutron scattering process is the result of a nuclear interaction depending on the spins of both the neutron and the target nucleus. This process can be taken into account by a single quantity, the scattering length $b_{i}$. The development of the $\left\langle b_{i} b_{j}\right\rangle$ term of Eq.4 leads to an expression where the scattered intensity is expressed in terms of coherent and incoherent contributions (Fig.4):

$$
\left(\frac{d^{2} \sigma}{d \Omega d \omega}\right)=\left(\frac{d^{2} \sigma}{d \Omega d \omega}\right)_{c o h}+\left(\frac{d^{2} \sigma}{d \Omega d \omega}\right)_{i n c}
$$

The terms of this equation are weighted by the neutron scattering $\sigma_{c o h}$ et $\sigma_{\text {incoh }}$ cross-sections:

$$
\begin{aligned}
\left(\frac{d^{2} \sigma}{d \Omega d \omega}\right)_{c o h} & =\frac{k_{1}}{k_{0}} \frac{\sigma_{c o h}}{4 \pi} S(Q, \omega) \\
\left(\frac{d^{2} \sigma}{d \Omega d \omega}\right)_{i n c} & =\frac{k_{1}}{k_{0}} \frac{\sigma_{i n c}}{4 \pi} S_{i n c}(Q, \omega)
\end{aligned}
$$

In these expressions, as they carry the physics of the system, the important terms are the coherent and incoherent dynamical structure factors, $S(Q, \omega)^{5}$ and $S_{\text {inc }}(Q, \omega)$, respectively. The cross-sections prefactors only indicate the fact that the technique used to assess them is neutron scattering. $S(Q, \omega)$ and $S_{i n c}(Q, \omega)$ obey the so-called sum rules:

$$
\begin{gathered}
\int_{-\infty}^{+\infty} S(Q, \omega) \cdot d \omega=S(Q) \\
\int_{-\infty}^{+\infty} S_{i n c}(Q, \omega) \cdot d \omega=1
\end{gathered}
$$

The coherent structure factor $S(Q)$ (Eq.8) is usually a $Q$ dependent quantity showing for example Bragg peaks for crystalline materials or halos for liquids. After integration over $\omega$, the incoherent dynamical structure factor is a $Q$-independent signal (Eq.9). In Small Angle Neutron Scattering (SANS) or diffraction experiments, it is therefore often labeled as a "background" and one tends to minimize its contribution. In what follows, we will show that this incoherent contribution is much more than a background: its dependence in energy carries key informations on the dynamics of the system. This feature is made crystal-clear by the use of the van Hove formalism [3].

\subsection{The van Hove formalism}

Let's consider a particles assembly and $G(r, t)$ a correlation function indicating the probability to observe a particle at a position $r$ at time $t$, provided that a particle $P$ stands at a position $r=0$ at time $t=0$. One can split $G(r, t)$ in a self, $G_{s}(r, t)$, and a distinct, $G_{d}(r, t)$ contributions:

$$
G(r, t)=G_{s}(r, t)+G_{d}(r, t)
$$

$G_{s}(r, t)$ is the probability that the initial particle $P$ stands at $r$ at time $t$, while $G_{d}(r, t)$ is the probability that it is a different particle. The van Hove formalism [3] establishes a direct connection between the correlation functions above and the scattered intensity:

$$
\begin{aligned}
S(Q, \omega) & =\frac{1}{2 \pi} \int G(r, t) e^{i(Q r-\omega t)} \cdot d r . d t \\
S_{\text {inc }}(Q, \omega) & =\frac{1}{2 \pi} \int G_{s}(r, t) e^{i(Q r-\omega t)} \cdot d r . d t
\end{aligned}
$$

\footnotetext{
${ }^{5}$ Note that, by convention, the coherent dynamical structure factor does write $S(Q, \omega)$ and not $S_{c o h}(Q, \omega)$
} 


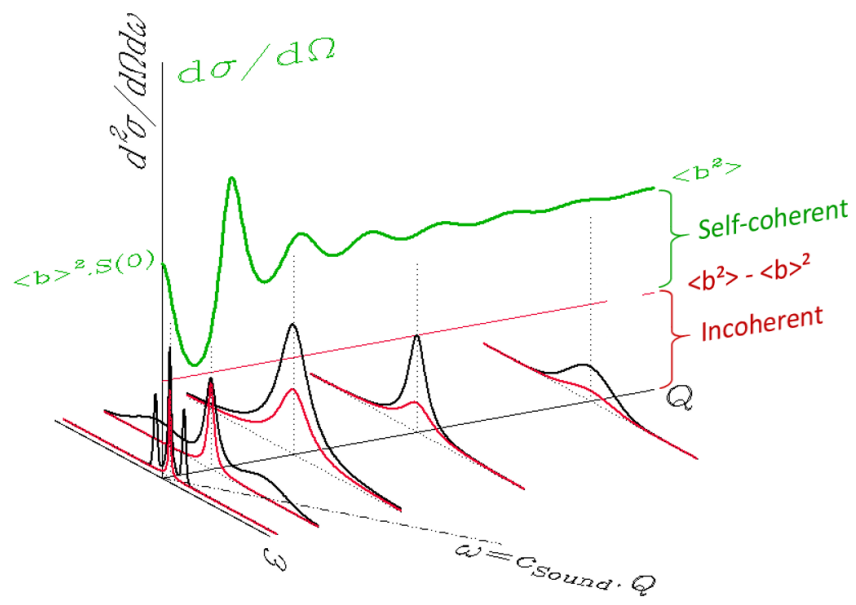

Figure 4. Schematic representation of the contributions to the neutron intensity scattered by a liquid sample in the quasi-elastic region. The coherent and incoherent inelastic contributions are shown as black and red lines on the basal $(Q, \omega)$ plane. After integration over energy, the incoherent inelastic contribution reduces to a flat background of intensity $\left\langle b^{2}>-<b\right\rangle^{2}$ (Eq.9). As, here, for the sake of clarity, we neglect the strong inelastic processes, the structure factor $S(Q)$ (Eq.6, green line), oscillates on top of this incoherent contribution, around the level of the self-coherent contribution value $<b^{2}>$. From [4].

- The coherent intensity is partly related to the "distinct" $G_{d}(r, t)$ term. It therefore accounts for a collective information. As it appears from equation 8 , for a coherent system, after integration in energy, the analysis of the scattered intensity provides informations on the structure of the system. One should note, that the "self" term also contributes to Eq.10 (terms $i=j$ of the array Eq.4). This so-called "self-coherent" contribution is a $Q$ independent signal, adding up to the incoherent scattering to define the high $Q$ limit of diffracted signals (see Fig.4). $S(Q)$ is the integral of the coherent dynamical structure factor over the energy, and the $Q$ Fourier transform of the total van Hove correlation function at $t=0$ :

$$
S(Q)=\int_{-\infty}^{+\infty} S(Q, \omega) \cdot d \omega=\int G(r, t=0) \cdot e^{-i Q r} \cdot d r
$$

If one now consider the dependence in energy of the coherent intensity, it is possible to assess collective dynamical processes like phonons for example.

- The incoherent intensity is only connected to the self contribution "self" $G_{s}(r, t)$. As this signal only carries an individual information, it does not give access to any direct information regarding the structure (Eq.9) of the system. Depending on the energy associated with the incoherent process, one defines:

- the elastic (i.e. $\hbar \omega=0)$ incoherent scattering: it can be used to access indirect dynamical information (mean-square displacements, confinement volume of a particle).

- the quasi-elastic (i.e. energy exchange of few meV) incoherent scattering: it can be used to measure self-diffusion coefficients, residence times in jump diffusion processes.

- the inelastic incoherent scattering: it can be used to perform vibrational spectroscopy. It can be used to extract the vibrational density of states i.e. the distribution in energy of the vibrational modes of the system. 
Coherence or incoherence of the scattered intensity are driven by the isotopic composition of the system. Specific devices (polarizers, analyzers) can be used to specifically access the coherent or the incoherent contribution of the scattered intensity. This can be performed, for example, on NSE instruments.

\subsection{Taking advantage of isotopic effects}

Experimentally, coherent and incoherent scattering can be used to distinguish individual and collective processes. The table 1 shows the values of the coherent and incoherent neutron cross-sections of most of the isotopes used in soft-matter experiments. The hydrogen atom shows an incoherent scattering

\begin{tabular}{|c|c|c|c|c|}
\hline \hline Element & $\begin{array}{c}b_{c o h} \\
(\mathrm{fm})\end{array}$ & $\begin{array}{c}\sigma_{c o h} \\
(\text { barn })\end{array}$ & $\begin{array}{c}\sigma_{\text {inc }} \\
(\text { barn })\end{array}$ & $\begin{array}{c}\sigma_{a b s} \\
(\text { barn })\end{array}$ \\
\hline $\mathrm{C}$ & 6.64 & 5.55 & 0.001 & 0.0035 \\
$\mathrm{~N}$ & 9.36 & 11.01 & 0.50 & 1.90 \\
$\mathrm{O}$ & 5.803 & 4.232 & 0 & 0.000191 \\
$\mathrm{~S}$ & 2.847 & 1.0188 & 0.007 & 0.53 \\
$\mathrm{H}$ & -3.7406 & 1.7583 & 80.27 & 0.3326 \\
$\mathrm{D}$ & 6.671 & 5.592 & 2.05 & 0.000519 \\
\hline \hline
\end{tabular}

Table 1. Coherent and incoherent scattering cross-sections of different elements and isotopes. The absorption cross-sections, $\sigma_{a b s}$, are indicated for an incident wavelength of $1.8 \AA$ [5].

cross-section 40 times higher than the one of the deuterium, its isotope. Also, by comparing the numerical values, it is clear that in hydrogenated compounds, the incoherent contribution of hydrogen largely dominates all the scattering contributions from the other atoms. The incoherent dynamic structure factor of such compounds can therefore be directly related to the individual dynamics of the sample's protons.

In the soft-matter and biology fields, specific deuteration can be used to minimize the detrimental contributions of specific moieties: typically the solvent and/or side-chains can be deuterated. In section 4.4, we also show that a mixture of hydrogenated and deuterated polymer chains can be used to access the dynamical contribution of a "single" chain.

\subsection{Derivation of $S_{i n c}(Q, \omega)$ for selected examples of dynamical modes}

In this section, we provide an overview, in both the time and energy domains, of how key typical dynamical modes can be apprehended in inelastic and quasi-elastic neutron scattering. This is also summarized on Fig.5.

\subsubsection{Long range translational and jump diffusion}

- Random walk diffusion (Fick's law)

The isotropic diffusion mechanism of a particle can be described as a cyclic phenomenon: following an interaction, with another particle by example, the particle is set in motion and travels a path $r_{\text {elem }}$ in space over a time $\tau$. Then, a new event changes the speed and direction of the particle so that the initial history of the particle (position, speed, direction) is lost. One can show that [7], if we observe 

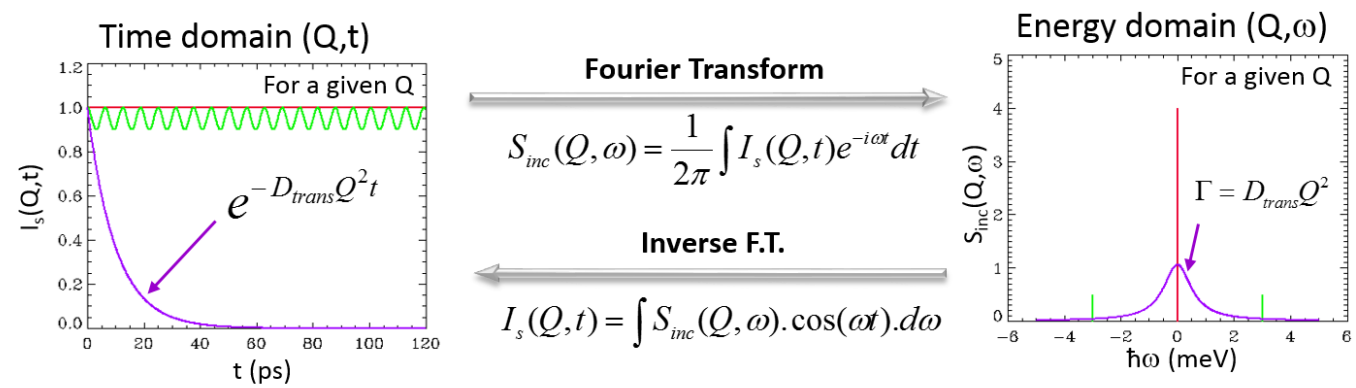

Figure 5. Schematic representation of the inelastic spectra of different dynamical modes. We consider the autocorrelation function $I_{s}(Q, t)$ of a particle in the $(Q, t)$ domain. The switch to the $(Q, \omega)$ domain is achieved by time Fourier Transform of $I_{s}(Q, t)$. By definition of an autocorrelation function, whatever the dynamics of the particle, its position at $t=0$ is known i.e. $I_{s}(Q, t=0)=1$. If the particle is immobile, whatever $t$, its position is also known i.e. $I_{s}(Q, t)=1$ (see red curve). The Fourier transform in time of this horizontal straight line is a dirac function in energy positioned at $\hbar \omega=0$. This physically corresponds to a situation where neutrons do not exchange any energy with the particle. One talks of "elastic scattering". If now a particle experiences a vibrational mode at frequency $\hbar \omega_{0}$, the autocorrelation function $I_{s}(Q, t)$ is a sinusoidal function of time. Its Fourier transform materializes as an elastic dirac along with two diracs centered at $\hbar \omega_{0}$ and $-\hbar \omega_{0}$. These two diracs of the inelastic zone of the spectrum $(\hbar \omega \neq 0)$ represents the simpler expression of $g(\omega)$, the vibrational density of states (see section 2.5.3). In the case of a particle experiencing a long-range translational diffusion (c.f. section 2.5.1), $I_{s}(Q, t)$ takes an exponential form (Eq.14). The corresponding dynamical structure factor is a Lorentzian line centered around $\hbar \omega=0$ with HWHM, $\Gamma$ (Eq.15). From [6].

the system at a scale larger than $r_{\text {elem }}$ and a long time compared to $\tau$, the probability of finding the particle at a position $r$ at time $t$ obeys the relation:

$$
\frac{\partial G_{s}(r, t)}{\partial t}=D_{t} \nabla^{2} G_{s}(r, t)
$$

where $D_{t}$ is the long range diffusion coefficient. With the boundary conditions $G_{s}(Q, 0)=\delta(r)$ and $G_{s}(r, \infty)=0$, the solution of this differential equation is:

$$
G_{s}(r, t)=\frac{1}{\left(4 \pi D_{t} t\right)^{\frac{3}{2}}} e^{-\frac{r^{2}}{4 D_{t} t}}
$$

Physically, this relationship means that the probability of knowing the position of the particle at a distance $r$ from its initial position is a Gaussian function of space $(r)$. The square of the standard deviation (quantifying "the spreading" of the curve) is directly proportional to time. The passage in the reciprocal space is made by space Fourier Transform:

$$
I_{S}(Q, t)=\frac{1}{2 \pi} \int G_{s}(r, t) \cdot e^{i(Q r)} \cdot d r=e^{-D_{t} \cdot Q^{2} \cdot t}
$$

The corresponding incoherent dynamic structure factor is obtained by time Fourier transform:

$$
S_{\text {inc }}(Q, \omega)=\frac{1}{\pi} \frac{D_{t} Q^{2}}{\left(D_{t} Q^{2}\right)^{2}+\omega^{2}}
$$


$S_{\text {inc }}(Q, \omega)$ is a Lorentzian line in energy with integrated intensity 1 (i.e. $\int_{-\infty}^{+\infty} S_{\text {inc }}(Q, \omega) \cdot d \omega=1$ ), of Half-Width at Half Maximum (HWHM) $\Gamma=D_{t} Q^{2}$. $\Gamma$ shows a linear dependence vs $Q^{2}$ with a slope $D_{t}$. It is referred as the so-called " $D Q^{2}$ law" (Fig.6).
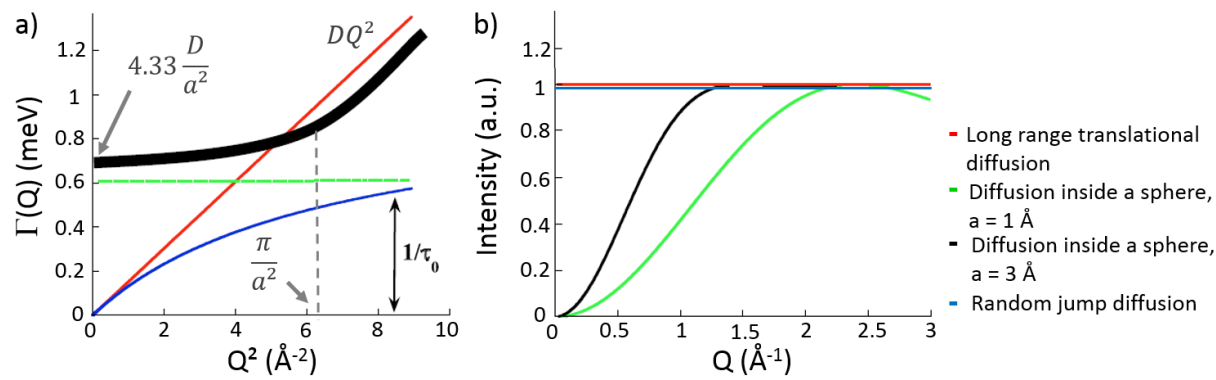

Figure 6. $Q$ dependence of the quasi-elastic HWHM and intensity (integrated in $\omega$ ) of selected dynamical processes. In red: long range translational diffusion (Fick's law or $D Q^{2}$ law, Eq.15). Blue: jump diffusion (Eq. 16). Green: reorientational diffusion (Eq.22). Black: diffusion within a confinement volume [7, 8].

\section{- Jump diffusion.}

In the situation where the $(Q, \omega)$ range used allows to access phenomena with spatial and temporal characteristics of the same order of magnitude than those of the elementary process leading to the diffusion, the conditions that led us to write Eq.12 is no longer valid. It is the case when working at large vector of diffusion. In the model of jump diffusion [7], it is assumed that between two events leading to diffusion, the particle remains on a given site for a time $\tau_{0}$, very long in front of the jump duration $\tau$. In this case, the dynamic structure factor writes:

$$
S_{\text {inc }}(Q, \omega)=\frac{1}{\pi} \frac{f(Q)}{f(Q)^{2}+\omega^{2}}
$$

$S_{\text {inc }}(Q, \omega)$ is therefore a Lorentzian line with a HWHM $f(Q)$ :

$$
f(Q)=\frac{D_{t} Q^{2}}{1+D_{t} Q^{2} \tau_{0}}
$$

At small $Q$, the $Q$ dependence of $f(Q)$ is similar to the one of the $D Q^{2}$ law. At large $Q, f(Q)$ leaves linear behavior to tend towards the asymptotic value of the reverse of the residence time $\tau_{0}$ (Fig.6).

\subsubsection{Diffusion in restricted geometry. The notion of EISF}

\section{- The concept of EISF.}

The autocorrelation function of the individual dynamics of a particle is described by the van Hove autocorrelation function.

At $\mathrm{t}=0, G_{s}(r, 0)=\delta(r)$. The "self" contribution is obtained by inverse Fourier transform: $I_{s}(Q, t)=\int G_{s}(r, t) \cdot e^{i Q r} \cdot d r$.

Let's consider that the particle is experiencing a perturbation at $\mathrm{t}=0$. After relaxation, at $t=\infty$, the 

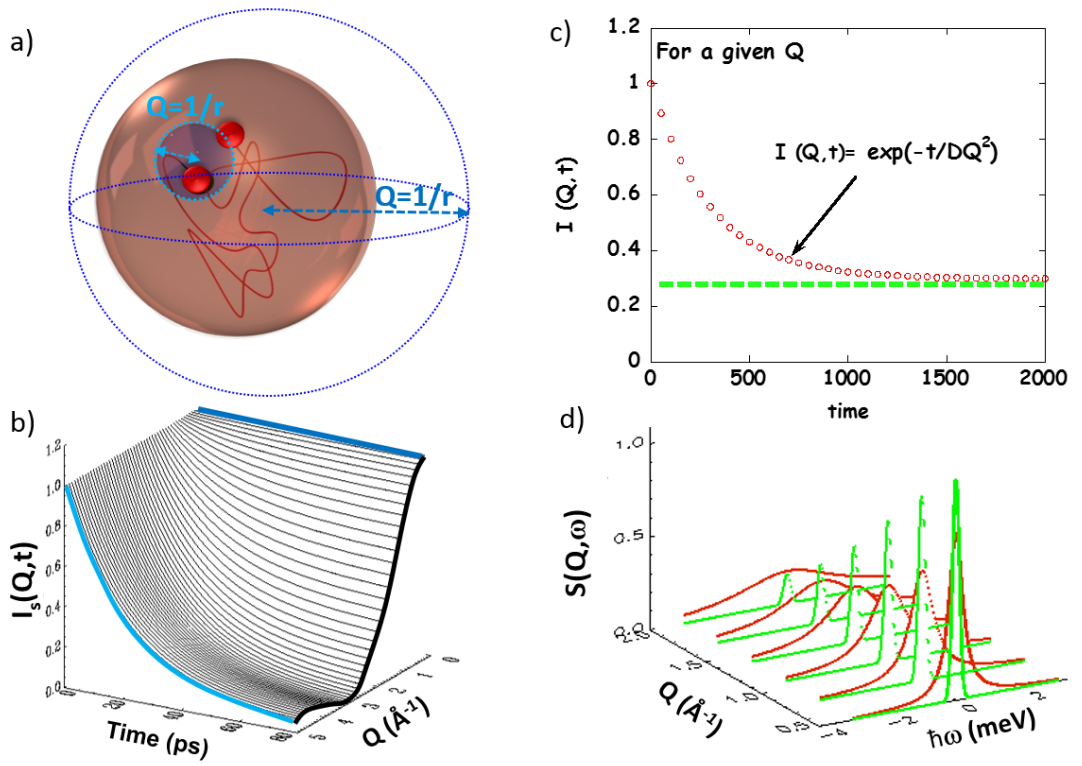

Figure 7. The Incoherent Elastic Structure Factor: EISF. a) Consider a particle (red dot) experiencing a diffusion process (trajectory in red) in a confinement volume (brown sphere). b) 3D representation of the evolution of the corresponding intermediate self scattering function $I_{s}(Q, t)$. Each curve represents the evolution as a function of time of $I_{s}(Q, t)$, at a given $Q$ value, i.e. at a scale $r \approx 2 \pi / Q$ of observation of the system. Whatever this scale, at $t=0$, by definition of an autocorrelation function, the position of the particle is known: $I_{s}(Q, t=0)=1$. At longer time, $(i)$ if we look at the system on a very local scale (light blue dotted sphere in (a)) i.e at large $Q$, the particle diffuses and informations about its position are quickly lost: we end-up at $I_{s}(Q, t=0) \approx 0$ (light blue line in (b)). (ii) If we observe the system from afar, i.e. on a much larger scale than the size of the confinement cage (blue dotted sphere in (a)), $1 / r$ is large so $Q$ is small), at any moment the position of the particle is known: it sits somewhere inside the confinement cage so $I_{s}(Q, t) \approx 1$ (blue line in (b)). c) Between these two extreme scales of observation, $I_{s}(Q, t)$ adopts an intermediate behavior, characterized by the presence of a plateau (in green). The curved defined by $I_{s}(Q, t=\infty)$ is a function of a single parameter $Q$ : this is nothing else than the form factor (Structure Factor) of the confinement volume (in black in (b)). Since the particle is subject to a diffusion process, next to the plateau, the time dependent contribution takes the form of Eq.14. It carries the information on the dynamics of the system. d) 3D representation of the evolution of the dynamic structure factor, $S_{\text {inc }}(Q, \omega)$, recorded on a ToF spectrometer. In the $(Q, \omega)$ domain, for each $Q$-value, the plateau and exponential of the $(Q, t)$ domain of (b) leads to an elastic peak of intensity $I_{E l}=I_{s}(Q, t=\infty)$ and a quasi-elastic intensity $\left(I_{Q u a s i}=1-I_{s}(Q, t=\infty)\right)$, respectively (cf. Fig.5). In the $(Q, t)$ domain, for each $Q$-value, the plateau is characterized by the elastic fraction of the signal. It is the same in the $(Q, \omega)$ domain: $E I S F=I_{E l} /\left(I_{E l}+I_{Q u a s i}\right)$. As it is derived from a plateau in the time domain of an autocorrelation function, hence the elastic intensity of an incoherent scattering, this form factor is called an Elastic Incoherent Structure Factor (EISF). From [6].

system is back to equilibrium, and the correlation with the position at $\mathrm{t}=0$ has been lost [7]:

$$
\begin{aligned}
I_{s}(Q, t=+\infty) & =\left\langle e^{-i Q r(0)} e^{-i Q r(t=+\infty)}\right\rangle \\
& =\left\langle e^{-i Q r(0)}\right\rangle\left\langle e^{-i Q r(t=+\infty)}\right\rangle \\
& =\left|\left\langle e^{-i Q r(t=+\infty)}\right\rangle\right|^{2} \\
& =\int G_{s}(r, t=+\infty) e^{i Q r} \cdot d r
\end{aligned}
$$


$I_{s}(Q, t=+\infty)$ is therefore the form factor of the region of space accessible to the particle (Fig.7). The dynamic incoherent structure factor can be decomposed in a time dependent quantity and a constant value with time, corresponding in the time domain to: $I_{s}(Q, t)$ and $I_{s}(Q, t=+\infty)$, respectively:

$$
S_{i n c}(Q, \omega)=I_{s}(Q, t=+\infty) \cdot \delta(\omega)+S_{i n c}^{q e}(Q, \omega)
$$

where $S_{i n c}^{q e}(Q, \omega)$ accounts for the quasi-elastic intensity noticeable around the elastic peak $\delta(\omega)$. The elastic peak intensity of an incoherent scattering spectrum of a particle subjected to a confined diffusion is therefore closely related to the form factor (structure factor) of the space of confinement of the particle. We talk about Elastic Incoherent Structure Factor (EISF). A more phenomenological description is given on Fig.7.

\section{- Experimental determination of the EISF.}

By integration of the dynamical structure factor at constant $Q$ :

$$
\begin{aligned}
\left.\int_{-\infty}^{+\infty} S_{\text {inc }}(Q, \omega)\right|_{Q \text { constant }} d \omega & =\int_{-\infty}^{+\infty}\left(\int_{-\infty}^{+\infty} I_{s}(Q, t) e^{-i \omega t} \cdot d t\right) \cdot d \omega \\
& =\int_{-\infty}^{+\infty} I_{s}(Q, t) \cdot \delta(t) \cdot d t \\
& =I_{s}(Q, 0)=1
\end{aligned}
$$

According to Eq.19 and Eq.20, $I(Q, \infty)$ is the elastic contribution of the total scattered intensity (elastic + quasi-elastic). Therefore, after fitting the total intensity with a model accounting for both the elastic and quasi-elastic contributions, the EISF is calculated according to:

$$
\operatorname{EISF}(Q)=\frac{I^{e l}(Q)}{I^{e l}(Q)+I^{q e}(Q)}
$$

where $I^{e l}(Q)$ and $I^{q e}(Q)$ are the elastic and quasi-elastic intensity, respectively. The comparison of this experimental value with a theoretical EISF derived from a model makes it possible to access to the geometry of a particle motion.

\section{- Rotational diffusion}

In the case of a particle diffusing freely over a sphere of radius $b$, with a good approximation [9], the incoherent dynamical structure factor writes

$$
S_{i n c}(Q, \omega)=B_{0}(Q) \delta(\omega)+\left[1-B_{0}(Q)\right] L\left(\omega, \Gamma_{r}\right)
$$

where $D_{R}$ is the rotational diffusion coefficient, $B_{0}(Q)=j_{0}(Q b)^{2}$ and $L\left(\omega, \Gamma_{r}\right)$ stands for a Lorentzian line with a HWHM $\Gamma_{r}=2 D_{R}$. At the opposite of the free (non-confined) diffusion: $i$ ) the particle being confined in a restricted portion of the space induces the existence of an EISF and hence the presence of an elastic peak in the expression of the dynamical structure factor, ii) $\Gamma_{r}$ is $Q$ independent. This is a property common to all the reorientational processes. 


\subsubsection{Vibrational dynamics}

\section{- Vibrational density of states and mean-square displacements.}

Unlike infra-red or raman spectroscopy, there is no selection rules for inelastic neutron scattering. Any accessible transitions in the energy range where the experiment is carried out, are allowed. We can therefore access a true weighted density of state. Note however that the intensity excitations strongly depend on the temperature of the system. The frequency distribution function of the vibrations (eigen modes, resonant frequencies ...) of a system is the so-called vibrational density of states (VDOS), $g(\omega)$. At a temperature T, the intensity scattered elastically by an assembly of $\mathrm{N}$ harmonic oscillators, of mass $\mathrm{M}$, experiencing various isotropic inelastic modes in three dimensions (of density of states $g(\omega))$ is proportional to:

$$
S(Q, \omega=0)=e^{-Q^{2}\left\langle u^{2}\right\rangle / 3} . \delta(\omega) \quad \text { with } \quad\left\langle u^{2}\right\rangle=\frac{\hbar}{6 M N} \int \frac{g(\omega)}{\omega} \operatorname{coth}\left(\frac{\hbar \omega}{2 k_{B} T}\right) \cdot d \omega
$$

For $\hbar \omega \gg 2 k_{B} T$, at high enough temperatures, $\operatorname{coth}(x) \simeq \frac{1}{x}$ Eq.23 simplifies:

$$
\left\langle u^{2}\right\rangle=\frac{3 k_{b} T}{M} \int \frac{g(\omega)}{w^{2}} \cdot d \omega
$$

In purely harmonic systems, the frequency of the vibrational modes are strictly independent of the temperature and therefore of the shape of $g(\omega)$. However, as the intensity is proportional to the population of the different modes, $g(\omega)$ is controlled by the temperature of the sample via the Bose population factor $n(\omega)$ (equivalent to the Maxwell-Boltzmann statistics at high temperature). Eq.23 shows that the vibrational modes (vibration of atoms around their position of equilibrium) induce a Gaussian loss of intensity, clearly observable at large $Q$ : this is the famous Debye-Waller effect existing also in X-ray diffraction. The scattered intensity in the inelastic zone of the spectrum gives access to $g(\omega)$. On the other hand, according to Eq.23, a fit of $\ln (S(Q, \omega \approx 0)) v s Q^{2}$ is an estimate of the total spatial extension induced by the different mode, $\left\langle u^{2}\right\rangle$ (Fig.8a). However, it should be noted that for a solid, essentially static, the neutrons mainly undergo an elastic diffusion and that the inelastic bands are much less intense than the elastic peak (ratio of the order of one to hundred). Fine measurement of the density of vibrational states, $g(\omega)$, demands an excellent signal-to-noise ratio. A deviation from the linearity of $\left\langle u^{2}\right\rangle v s T$ is characteristic of the appearance/disappearance of dynamical (diffusional for example) and/or vibrational modes. In practice, the temperature where the quasi-elastic signal effectively is determined by performing a so-called "Elastic Scan": the sample temperature is submitted to a temperature ramp (typically $0.5 \mathrm{~K} / \mathrm{min}$ ) and at each temperature the scattering is recorded for a short time (typically 5 minutes). As the scattering is generally intense around the elastic peak, the uncertainty of the slope of the $\ln (S(Q, \omega \approx 0)) v s Q^{2}$ linear fit is moderate and a curve showing the temperature evolution of of $\left\langle u^{2}\right\rangle$ with several hundreds of point can be plotted (see Fig.8b). Once the temperature range of the quasi-elastic signal has been determined, one can perform long scans at selected temperature to record the quasi-elastic signal with a good statistics (see section 4.2).

\section{- Connection with thermodynamics}

According to Klug et al. [11] the vibrational heat capacity can be accurately estimated below $100 \mathrm{~K}$ from the inelastic neutron spectra (INS) using the harmonic oscillator model

$$
C_{v}=R \int \frac{g(\omega)\left(\hbar \omega / k_{B} T\right)^{2} \exp \left(\hbar \omega / k_{B} T\right)}{\left(\exp \left(\hbar \omega / k_{B} T\right)-1\right)^{2}}
$$



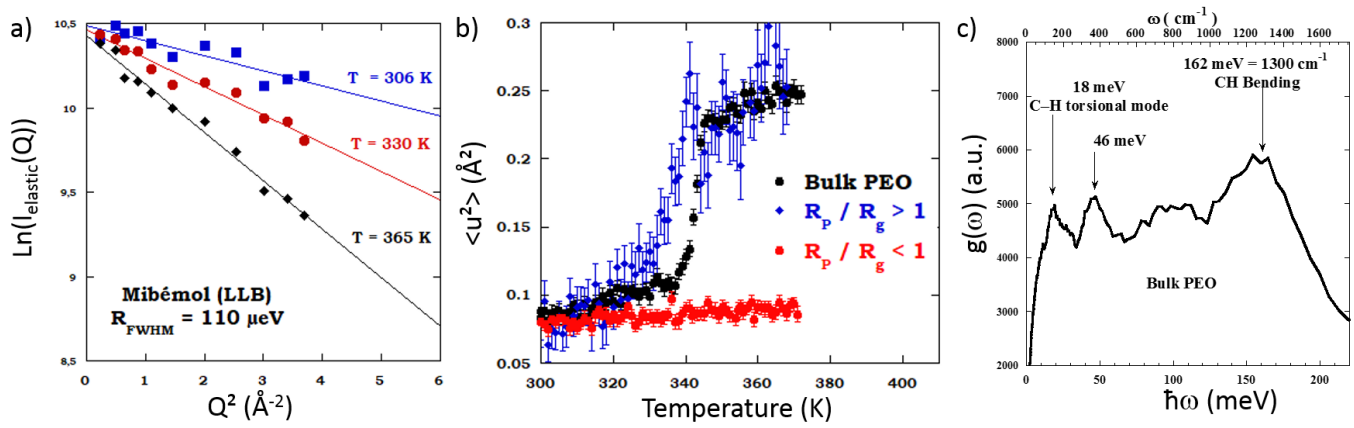

Figure 8. a) $Q$ dependent Gaussian loss of elastic intensity $I_{e l}$ of $35 \mathrm{~kg} / \mathrm{mol}{ }^{h} P E O$ at selected temperature. b) Practical result of an "elastic scan" (see text) showing the temperature evolution of $\left\langle u^{2}>\right.$ obtained from a linear fit of $\ln \left(I_{e l}\right) v s Q^{2}$, in the [300-373 K] r ange. For bulk PEO, the clear jump of $<u^{2}>$ at $331 \mathrm{~K}$, indicates the sample melting. Above this temperature, diffusive motions come into play, and are detected as a broadening of the the quasi-elastic line of the $S_{\text {inc }}(Q, \omega)$ spectrum. The plot b) illustrate the influence of confinement on PEO dynamics. The confining material is an Aluminum Oxyde Membrane (AAO, see Fig.16). On can define two characteristic lengths in the system: $R_{P}$, the $\mathrm{AAO}$ pore diameter, and $R_{g}$, the radius of gyration of the polymer chain (for a $35 \mathrm{~kg} / \mathrm{mol}$ PEO chain, $R_{g}=69 \pm 1 \AA$ ). Depending on the ratio of $R_{P} / R_{g}$, one can detect $i$ ) a clear downshift of the melting point (Gibbs-Thomson effect [10]) for $R_{P} / R_{G}>1$ and ii) a glass-like arrest of the polymer dynamics for $R_{P} / R_{G}<1$. c) Vibrational density of states, $g(\omega)$ of a bulk PEO measured on a spallation source (inverted geometry spectrometer Fig.14). Note that the high incident energy of incoming neutrons allows to reach high neutron energy loss.

where $C v$ stands for the isochoric molar heat capacity, $\mathrm{R}$ is the gas constant, and $k_{B}$ is the Boltzmann's constant. The anharmonic part of the specific heat associated with the thermal expansion $C p C v=T V \alpha^{2} / \kappa_{T}$ (where $V$ is the molecular volume, $\alpha$ is the volume thermal expansion, and $\kappa_{T}$ is the isothermal compressibility) is estimated to be negligible (for ice: $0.025 \mathrm{~J} \mathrm{~mol}^{-1} \mathrm{~K}^{-1}$ at $100 \mathrm{~K}$ [12]). Thus the isobaric heat capacity, $\mathrm{Cp}$, is virtually indistinguishable from the isochoric specific heat Cv. Above $100 \mathrm{~K}$, the vibrational heat capacity of ice becomes noticeably anharmonic [11] and deviations of the calculated heat capacity from the experimental values are expected. The theory is exact for simple crystals and valid in a good approximation for amorphous systems with dominant incoherent scattering. In biomolecules, on average, half of the atoms are protons and their roughly homogeneous distribution reflects the collective dynamics. Also as neutron scattering originate from nuclear interactions, no selection rule applies, so that $g(\omega)$ can be considered as a genuine VDOS [13].

\subsubsection{The total dynamical structure factor}

The total dynamical structure factor is the convolution of the different elemental structure factors described in the previous sections:

$$
S_{i n c}(Q, \omega)=S_{i n c}^{\text {trans }}(Q, \omega) \otimes S_{i n c}^{r o t}(Q, \omega) \otimes S_{i n c}^{v i b}(Q, \omega)
$$

The vibrational contribution is the sum of an elastic and an inelastic contribution due to the internal vibrations of the molecule $\left(S_{i n c}^{V}(Q, \omega)\right)$ and the lattice vibrational modes $\left(S_{\text {inc }}^{L}(Q, \omega)\right)$ :

$$
S_{i n c}^{v i b}(Q, \omega)=e^{-2 W(Q)}\left(\delta(\omega)+S_{i n c}^{V}(Q, \omega)+S_{i n c}^{L}(Q, \omega)\right)
$$


The inelastic part $S_{i n c}^{V}(Q, \omega)$ is composed of a collection of $\delta$ functions each centered at frequencies corresponding to each molecular vibrational modes. The contribution $S_{i n c}^{L}(Q, \omega)$ can be more complex, but can usually be taken into account by a flat background in the quasi-elastic region of the spectrum. In isotropic samples, it is possible to perform a powder average and the Debye-Waller factor writes:

$$
e^{-2 W(Q)}=e^{-Q^{2}\left\langle u^{2}\right\rangle / 3}
$$

$\langle u\rangle^{2}$ is the mean-square displacement of the atoms undergoing vibrational modes. The convolution product in Eq.26 simplifies:

$$
S_{\text {inc }}(Q, \omega)=e^{-Q^{2}\langle u\rangle^{2} / 3}\left(S_{\text {inc }}^{\text {trans }}(Q, \omega) \otimes S_{i n c}^{r o t}(Q, \omega)+S_{\text {inc }}^{I}(Q, \omega)\right)
$$

where $S_{i n c}^{I}(Q, \omega)$ is an inelastic term due to the convolution of $S_{i n c}^{V}(Q, \omega)$ and $S_{\text {inc }}^{L}(Q, \omega)$ by $S_{i n c}^{r o t}(Q, \omega) \otimes S_{i n c}^{\text {trans }}(Q, \omega)$. A detailed discussion of the approximations leading to this results can be found at page 66 of the reference [7]. In the quasi-elastic region of the spectrum, this equations reduces to:

$$
S_{\text {inc }}(Q, \omega)=e^{-Q^{2}\left\langle u^{2}\right\rangle / 3}\left(S_{\text {inc }}^{\text {trans }}(Q, \omega) \otimes S_{\text {inc }}^{r o t}(Q, \omega)\right)+\mathcal{B}(Q)
$$

In all cases, the time reversibility is not considered i.e. $I(Q, t)=I(Q,|t|)$, and the auto-correlation functions are considered as even and the corresponding Fourier Transforms are real.

\subsection{Aspects related to the sample temperature}

Let's consider a neutron with incident energy $\hbar \omega_{0}$ interacting with an assembly of harmonic oscillators at temperature $T$, i.e. with an average energy $E=k_{B} . T$ :

- If $\boldsymbol{k}_{\boldsymbol{B}} \boldsymbol{T} \gg \boldsymbol{\hbar} \boldsymbol{\omega}_{0}$ : this is a situation where the incident neutron is a cold particle coming in interaction with a hot medium. A significant fraction of the oscillators are in an excited state so the incident neutron has a large probability to gain energy upon interaction with the sample. The probability that the neutron loses energy is not zero, however, it can not lose more energy than it carries. The maximum energy transfer is then limited to $+\hbar \omega_{0}$.

- If $\boldsymbol{k}_{\boldsymbol{B}} \boldsymbol{T}<<\boldsymbol{\hbar} \boldsymbol{\omega}_{\mathbf{0}}$ : this is the reverse situation, where the incident neutron is a hot particle coming in interaction with a cold medium. The sample excited levels are poorly or not populated. It is thanks to the energy of the neutron that they will be populated (hence the neutron will be colder after the interaction). In this case, it is the initial energy of the neutron that limits the maximum observable energy transfer and not the temperature of the sample.

The considerations above explains that at a given scattering vector $Q$, the observed intensity is a function of $\omega$ but also the temperature, $T$, of the sample. This can be formalized using the Bose population factor: $n=1 /\left(e^{-\hbar \omega / k_{B} T}-1\right)$ :

$$
\left(\frac{d^{2} \sigma}{d \Omega d \omega}\right)=\frac{k_{1}}{k_{0}} \frac{1}{4 \pi} \cdot n_{B}(\omega, T) .\left[\sigma_{c o h} . S(Q, \omega)+\sigma_{i n c} . S_{i n c}(Q, \omega)\right]
$$

with $n_{B}(\omega, T)=n+1$ if $\omega<0$, and $n_{B}(\omega, T)=n$ if $\omega>0$. One can note that $2 n+1=\operatorname{coth}\left(\hbar \omega / 2 k_{B} T\right)$. These considerations on the $\hbar \omega_{0} / k_{B} T$ ratio have important consequences on the choice of the experimental conditions and on the type of spectrometer, to optimize the observation of an excitation (see sections 3.2.2 and 3.2.3). 


\section{Instrumentation}

Two types of neutron sources exist: spallation sources that deliver a pulsed beam and continuous sources (reactors). In a schematic way, in both cases, the wavelength distribution $\Phi(\lambda) v s \lambda$ of the beams delivered is characterized by a maxwellian contribution of wavelength $\lambda>0.5 \AA$. On pulsed sources, $\Phi(\lambda)$ shows, with respect to continuous sources, an important additional contribution (from order of $50 \%$ of the total flux) of the form $\Phi(\lambda) \approx 1 / \lambda$ so-called ephithermal contribution. This strong profile of very energetic neutrons allows (i) to access the intramolecular vibration dynamics at high energy $\left(\hbar \omega>1000 \mathrm{~cm}^{-1}\right)$ and at very low temperature, and (ii) to measure concomitantly dynamical (quasi-elastic) events and structural properties (small angle scattering and diffraction).

All types of inelastic neutron spectrometers have been used to study the dynamics of macromolecules (polymers and proteins). Next to successful studies of the collective dynamics of lipidic membranes [14] one can hardly cite studies taking advantage of the potentialities of three-axis spectrometers in the field of soft-matter. We have therefore made the choice to focus here on inelastic spectrometers using the time-of-flight technique. In all cases the central part of the instrument consists of the sample. We then distinguish the primary spectrometer (before the sample) and the secondary (after the sample). The functions of primary and secondary spectrometers vary by type of technology used: direct or inverted geometry. In both cases, the use of speed diagrams allows to easily understand the operation of a spectrometer (Fig.12-14).

\subsection{Principle of the time-of-flight technique}

Fig.9 describes the principle of a neutron inelastic time-of-flight measurement. The incident neutrons of a monochromatic beam with energy $E_{i}$, are scattered by the sample with an energy $E_{f}$ (Eq.1) in a direction oriented at an angle $\phi=2 \theta$ of the incident beam. Over this process the neutrons experience a momentum transfer, $Q$, and an energy transfer, $\hbar \omega$ (Eq.2) with the sample ${ }^{6}$. Eq. 2 shows the conservation laws of momentum and energy.

The key step in the determination of $S(Q, \omega)$ by the time-of-flight methods is to measure the energy of each scattered neutron, by evaluating its travel time (time of flight), t, over a known distance. In the context of the example presented here, this is a simple measurement of $v_{f}$, the velocity ${ }^{7}$ of the scattered neutron, over the sample-detector distance $L_{S D}$.

$$
v_{f}=\frac{L_{S D}}{t}
$$

in other terms, the ToF spectrum:

$$
\frac{d^{2} \sigma}{d \Omega d t}
$$

is the measurement, by unit of solid angle $d \Omega$ of the arrival time distribution function of the arrival times $t=n \Delta \tau$ ( $\Delta \tau$ is the sampling time unit) of the neutrons onto the detector (Fig.9). One can show [2] that the differential cross-section by energy $d \omega$ and solid angle $d \Omega$ units is directly connected ${ }^{8}$ to the dynamical structure factor $S(Q, \omega)[2]$ :

$$
\frac{d^{2} \sigma}{d \Omega d \omega}=N \frac{k_{f}}{k_{i}} \frac{\sigma}{4 \pi} S(Q, \omega)
$$

\footnotetext{
${ }^{6}$ The signs of $Q$ et $\hbar \omega$ are fixed by convention.

${ }^{7}$ Key formulas and orders of magnitudes: the energy of neutrons of wavelength $\lambda$ is $E[\mathrm{meV}]=81.79 /(\lambda[\AA])^{2}$. The time-of-flight, $\tau$, over a distance $\mathrm{D}$, for neutrons of wavelength $\lambda$ is $\tau[\mu \mathrm{s}]=252.8 . D[\mathrm{~m}] . \lambda[\AA]$ : neutrons of $5 \AA$ spend $1.26 \mathrm{~ms}$ to fly over a distance of 1 meter.

${ }^{8}$ Here one disregards the existence of coherent and incoherent cross section to consider a single global cross section $\sigma$.
} 


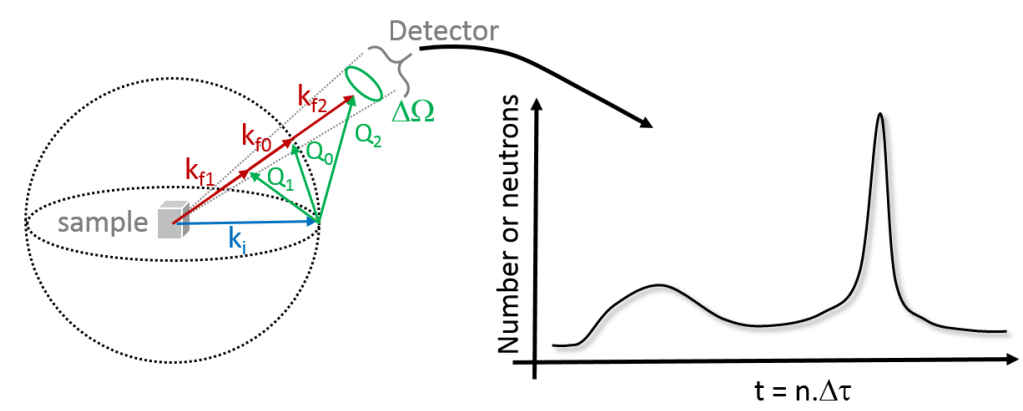

Figure 9. Schematic diagram of an inelastic measurement by time-of-flight: at an arbitrary origin of the time, a sample is briefly illuminated by a neutron burst. The distribution of the arrival time of the scattered neutrons at the detector equipped with an internal clock is then recorded. The so-called time-of-flight spectrum $\frac{d^{2} \sigma}{d \Omega d t}$ is shown here in black. The time scale is discretized into a succession of $\mathrm{n}$ constant time channels $t=n . \Delta \tau$. As the neutron-sample interaction is generally inelastic, spatial and energy quantities are linked by Eq.3. The three possible configurations are represented: $\left\|\vec{k}_{f_{1}}\right\|<\left\|\vec{k}_{i}\right\|$ : inelastic scattering with loss of energy of the neutrons (Stokes), $\left\|\vec{k}_{f_{0}}\right\|=\left\|\vec{k}_{i}\right\|:$ elastic scattering, $\left\|\vec{k}_{f_{2}}\right\|>\left\|\vec{k}_{i}\right\|:$ inelastic scattering with energy gain of the neutron (antiStokes). Adapted from [15].

By writing the expression 33 in the form:

$$
\frac{d^{2} \sigma}{d \Omega d t}=\frac{d \omega}{d t} \frac{d^{2} \sigma}{d \Omega d \omega}
$$

and considering that $E_{f}=\frac{1}{2} m_{n} v_{f}^{2}$, and by combining Eq.1, 32 and 34, one can writes:

$$
\frac{d^{2} \sigma}{d \Omega d t}=\frac{N \sigma}{h \sqrt{E_{i}}}\left(\frac{m_{n}}{2}\right)^{\frac{3}{2}} \frac{L_{S D}^{3}}{t^{4}} S(Q, \omega)
$$

The ToF spectrum is therefore directly related to the dynamical structure factor, but on the raw data it appears "deformed" by the term in time of flight to the power 4 . This can have important consequences on the appearance of the raw spectra (Fig.10). Since the weight of this term in $1 / t^{4}$ becomes significant when $t$ is large, the very precise subtraction of the electronic background, clearly detected on the first time channels ( $\mathrm{n}$ from 1 to about 10), is essential.

\subsection{Implementation}

The spectrometers of the so-called "ToF-ToF" technology are the ones which make it possible to illustrate the implementation of an inelastic neutron scattering experiment in the simplest way. The two essential steps of the measurement are the monochromatization of the incident beam and the energy analysis of the scattered beam. Both steps use the same principle: the energy discrimination of neutrons according to the time spent to travel a known distance (generally in the order of a few meters). 


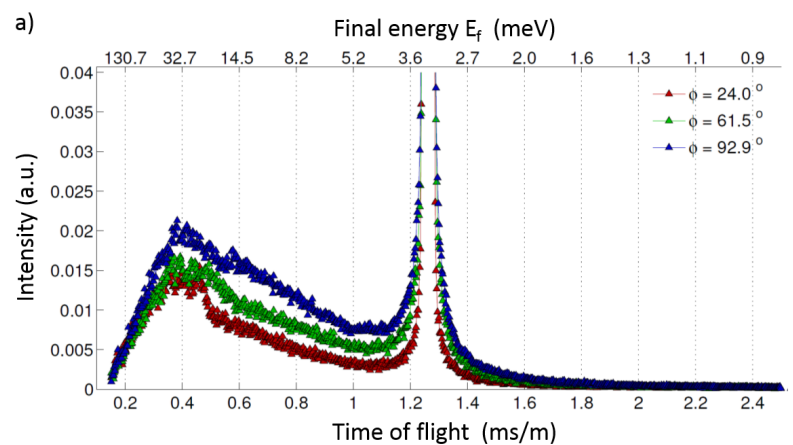

b)

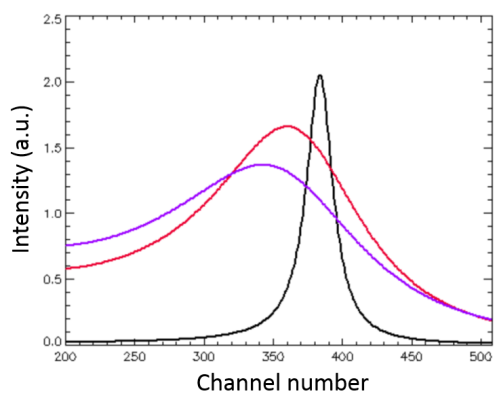

Figure 10. a) Example of a "raw" ToF spectrum. The abscissa axis is in ToF (the ToF scale, $t$, is proportional to the channel-width $\Delta: t[\mu s]=n . \Delta \tau[\mu s]$, where $n$ is a so-called channel number). Additionally, this scale has been here divided by the sample to detector distance $(4 \mathrm{~m})$. The corresponding energy scale is also shown (5 $\AA$ A incident neutrons) on top of the plot. Note that the scale is linear in time but not in energy. b) Several Lorentzian dynamical structure factor $S(Q, \omega)=\frac{\Gamma}{\pi} /\left(\Gamma^{2}+\omega^{2}\right)$ plotted in a channel number scale. All these quasi-elastic signals are centered at zero energy transfer $(\hbar \omega=0)$, corresponding here to the elastic channel $n_{\text {Elastic }}=385$. Three Lorentzians with $\Gamma$ values $0.05,0.3$, and $0.4 \mathrm{meV}$ are shown in black, red and blue respectively. Based on its HWHM, $\Gamma$, the raw spectrum may appear to be an inelastic signal, i.e. showing an offset with respect to the elastic channel. This is a consequence of the $t^{4}$ term of Eq.36.

\subsubsection{Monochromatisation}

A first chopper ${ }^{9}$ equipped with a single slot, rotating at the speed $\Omega_{c}$, "chops" the white incident beam ${ }^{10}$ in short-time pulses with a frequency $v[\mathrm{~Hz}]=\Omega_{c}[\mathrm{rpm}] / 60$ (Fig.11). At a distance $L_{12}$, a second chopper, identical to the first one, but out of phase by an $\Phi$ angle, reproduces this chopping operation of the beam with a time offset $\delta[s]=\Phi / v[\mathrm{~Hz}]$ modulo $1 / v$. Choosing the value of $\Phi$ allows to select the desired wavelength. As they are the ones that travel the distance $L_{S D}$ in a time $\delta$, only the neutrons with wavelength

$$
\lambda_{i}=\frac{h}{m_{n}} \frac{1}{L_{S D}} \frac{\Phi}{2 \pi v_{c}}
$$

are transmitted by the second chopper. The others ones are absorbed.

\section{- Analysis:}

In practice, the instant, $t_{0}$, the beam reaches the second chopper, defines the origin of the measurement times (Fig.11): the internal clock of the detector is started. The neutrons scattered by the sample are recorded according to their arrival time on the detector. It is the probability of an energy exchange between the neutron and the sample, described by the dynamic structure factor $S(Q, \omega)$, that spread, over the sample-detector distance $L_{S D}$, the neutron arrival time on the detectors. The neutrons that have gained energy arrive first at the detector, followed by elastically distributed neutrons arriving in the elastic channel (Fig.12). Finally, the neutrons having lost energy to the sample arrive last.

\footnotetext{
${ }^{9} \mathrm{~A}$ chopper is a neutron absorbing disk rotating in a continuous beam in which a or multiple slits have been made. The incident neutrons are absorbed by the chopper except when the slits passes in front of the beam. This system induces a "chopped" beam.

${ }^{10}$ By analogy with light, we denote by white beam a beam presenting an extended spectrum in wavelengths and therefore a distribution of energies. A monochromatic beam is composed of a single wavelength. See the section 3.3 for consideration on uncertainty on the wavelength determination and the underlying notion of resolution in energy of a spectrometer.
} 


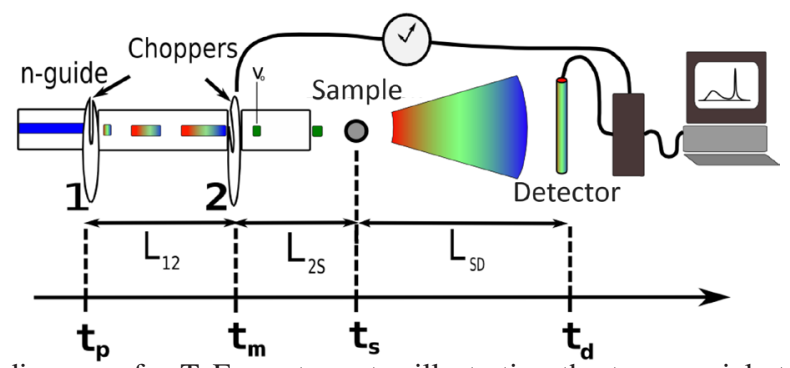

Figure 11. Schematic diagram of a ToF spectrometer illustrating the two crucial steps of the measurement: the monochromatization of the white (see 3.2.1) incident beam, then the time of flight analysis at the detector (details in section 3.2). For the sake of simplicity a single detector is represented. On a real spectrometer, the measurement in time of flight is performed simultaneously on several hundred detectors. From [15].

a)

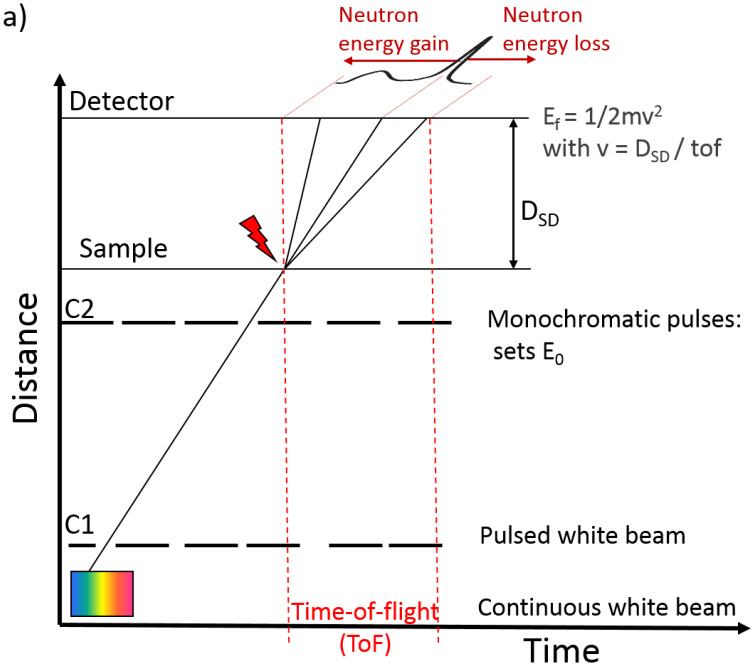

b)

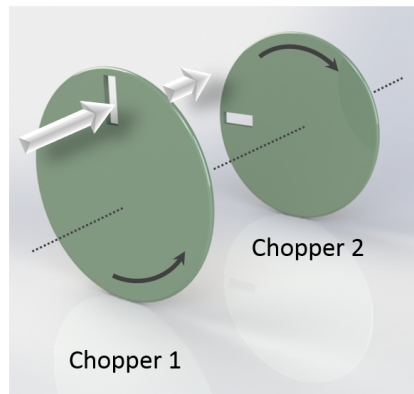

Figure 12. Time-distance diagram. This figure implements the so-called representation "Time-distance diagram". The time is plotted on the abscissa and the distances on the y-axis. A neutron of energy E i.e. with speed $v=\sqrt{2 E / m_{n}}$, is represented by a line with a slope $1 / v$. a) General principle: case of the direct geometry (the inverted geometry principle is shown on Fig.14. To perform a direct geometry ToF measurement, it is necessary to extract pulses of monokinetic neutrons, from a white beam (see 3.2.1). In principle, three devices are necessary: two choppers and a detector with an internal clock. From the continuous white beam (multicolored square) the first chopper (C1) produces a pulsed white beam. A phase adjustment $\varphi$ of the second chopper (C2) extracts from the white beam issued from $\mathrm{C} 1$ the neutrons having the desired wavelength (energy $E_{0}$ ). When these neutrons reach the sample, the detector clock is triggered. By interaction with the sample, the neutrons gain energy (speed increase, so also the slope on the graph), lose energy (decrease of speed, so decrease of the slope) or undergo an elastic interaction ( $\hbar \omega=0$, no change of slope). Then, they continue their trajectory until they reach the detector D located at a distance $D_{S D}$ from the sample. At every ToF moment (in each time channel), the number of neutrons arriving on the detector is recorded. The energy of each neutron having experienced a "time of flight", ToF, over the SD distance is $E_{f}=1 / 2 . m_{n}\left(D_{S D} / T o F\right)^{2}$. They have undergone an exchange of energy, $\hbar \omega=E_{i}-E_{f}$. An elementary derivation allows to switch from the ToF spectrum recorded on the detector to $S(Q, \omega)(\mathrm{Eq} .36)$. 


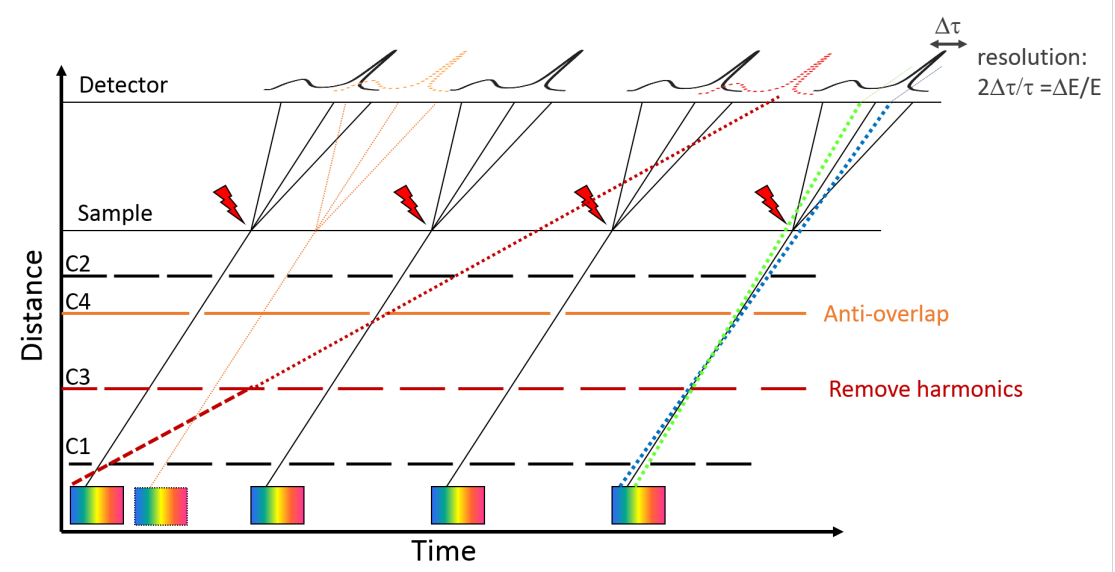

Figure 13. Time-distance diagram: direct geometry. In practice, to be usable, the system requires a little sophistication. A third chopper $\mathrm{C} 3$ acts as an anti-harmonic filter: it prevents neutrons having an integer submultiple speed not to reach the sample (red dots). A fourth and last chopper (called anti-overlap chopper), C4, having an integer sub-multiple rotational speed of the other choppers allows to space-out in time the pulses arriving on the sample. This ensures that the fastest neutrons of the $n+1$ pulse scattered by the sample can not be cought-up with the slowest neutrons of the pulse $n$. The window opening time (in front of the neutron guide) being a defined value (not infinitely small), there is an uncertainty of the order of a few percent on the neutron energy selected by the choppers system. Suppose a purely elastic sample-neutron interaction $\hbar \omega \approx 0$. The uncertainty on the speed of the incident neutrons produces an uncertainty about their arrival time (green/blue curves). The elastic peak is not infinitely thin, but has an intrinsic width $\Delta \tau$ : this is the energy resolution of the instrument. The higher speed the choppers, the shorter the window opening time in front of the guide. The uncertainty about the neutron departure time decreases but also the flux. One have always to compromise between flux and resolution.

\subsubsection{Direct geometry time-of-flight instruments: The chopper spectrometers}

In this type of technology, both the energies of the incident and of the scattered neutrons are selected by ToF.

\section{- Compromise between flux and resolution:}

One of the causes of the finite energy resolution, $\Delta E$, of the ToF spectrometers is the existence of a wavelength distribution, $\Delta \lambda$, at the detectors (Fig.13): $\Delta E / E=2 \Delta \lambda / \lambda=2 \Delta \tau / \tau$ where $\tau$ is the time of flight of neutrons and $\Delta \tau$, the associated time distribution. The most natural solution for reaching a resolution in energy of a few percent is to limit the distribution in incident neutron energy. This is the strategy adopted in the design of spectrometers with 2 choppers. The price to be paid for the gain in resolution is the low flux at the sample level. Also, these spectrometers can only occupy a position at the end of a guide.

\subsubsection{Inverted geometry time-of-flight instrument}

There is an alternative to choppers instruments technology: the so-called "inverted" geometry (Fig.14). The principle is to illuminate the sample with a white beam, and to detect by Bragg reflection on a crystal, the neutrons scattered with a specific energy. In Eq.3, the fixed parameter is $\hbar \omega_{1}$ and $\hbar \omega_{0}$ takes different values. This technology is particularly suited for pulsed sources that have a 
very wide spectrum of incident wavelengths, from 0.01 to $20 \AA$. With this type of spectrometer, it is possible to measure excitations of several hundred meV at very low temperatures (see discussion 2.6).

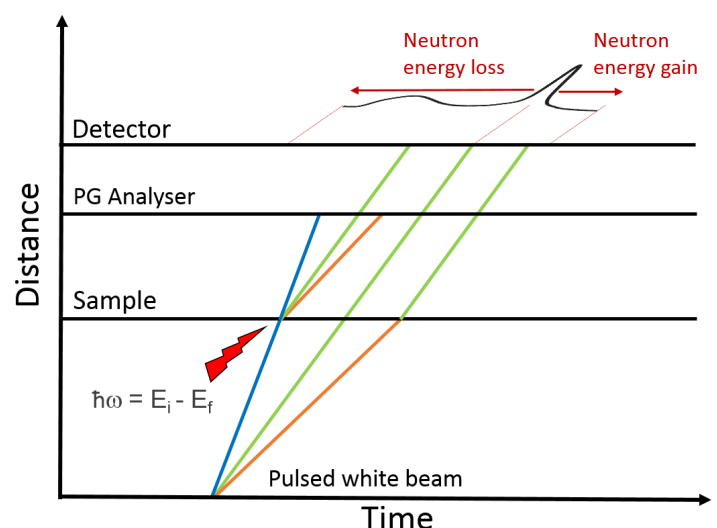

Figure 14. Time-distance diagram: inverted geometry. The sample is illuminated with a white (see 3.2.1) neutron beam. Only the neutrons scattered with a selected final energy (filtered by Bragg reflection on a monochromator) are sent to the detectors. While the experimental strategy is different (white vs monochromatic incident beam) for both the direct (Fig. 12) and indirect geometry, the recorded information is the probability for a neutron to be scattered at a specific $Q$ value with an energy exchange $\omega$ : this is the dynamical structure factor $S(Q, \omega)$.

-An advantage: The simultaneous measurement of the structure and of the dynamics

It is possible to have in the ToF base of the spectrometer, a detector without interposing the analyzer that selects the final energy $\hbar \omega_{1}$. The neutrons scattered by the sample arriving in this detector, located at a diffusion angle $2 \theta$, can be used to perform a diffraction experiment i.e. to measure ${ }^{11} I(Q)$ vs $Q$. Some detectors can therefore be used in diffraction simultaneously with detectors that retain the classical operation of energy analysis.

\subsection{Experimental energy resolution effects and consequences}

As a first approximation, ${ }^{12}$ the signal $S(Q, \omega)_{\text {exp }}$ measured by a spectrometer is the convolution product of the theoretical signal $S(Q, \omega)_{\text {theo }}$ (which would be measured if the device was infinitely resolutive) by an experimental resolution $R(Q, \omega)$ (Fig.15):

$$
S(Q, \omega)_{\exp }=S(Q, \omega)_{t h} \otimes R(Q, \omega)
$$

Therefore, by inverse Fourier Transform and applying the convolution theorem:

$$
I(Q, t)_{\exp }=I(Q, t)_{t h e o} \cdot R(Q, t)
$$

\footnotetext{
${ }^{11} Q=\frac{4 \pi}{\lambda} \sin (\theta)$ : contrary to the "standard case", here $\theta$ is fixed and $\lambda$ varies.

${ }^{12}$ When one talks about energy resolution of a spectrometer this is the "elastic" resolution. In reality the resolution is a function of the energy transfer. On a ToF chopper spectrometer, the resolution is proportional to the scattered neutron energy. Eq.38 is only a valid approximation in the quasi-elastic region.
} 
where $R(Q, t)$ is the inverse Fourier Transform of $R(Q, \omega)$.

Generally, $R(Q, \omega)$ is experimentally determined (Fig.13) by the measurement of the scattering spectrum of a vanadium sample (this is a metal, hence a purely elastic scatterer below its melting point of $1910^{\circ} \mathrm{C}$ ) having the same geometry than the sample. The vanadium being an incoherent scatterer, it also provides information on the detector efficiency. For relaxational or Gaussian processes, the time-energy equivalence ${ }^{13}$ writes:

$$
\Delta E[m e V]=\frac{0.658}{\Delta t[p s]}
$$

These resolution effects imply that in neutron scattering, the observed physical phenomena and their associated quantities (distances, correlation times) are only valid at the resolution of the spectrometer (i.e. within the time (energy) / space $(Q)$ domains accessible experimentally).
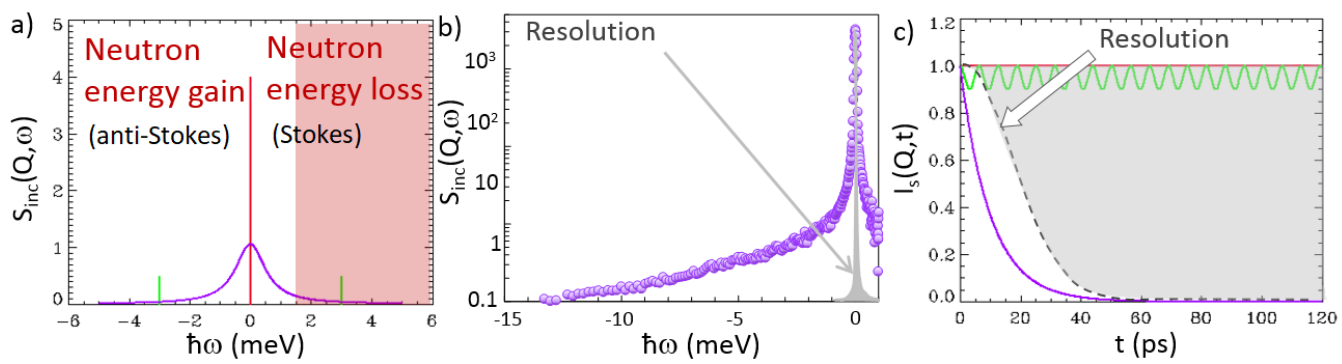

Figure 15. Theoretical neutron scattering spectrum and experimental limitations. a) The maximum energy a neutron can provide to the sample is limited by its own incident energy $\hbar \omega_{0}$. The red part of the neutron energy loss side is therefore inaccessible to neutrons with energy $\hbar \omega_{0}$. b) Experimental $S(Q, \omega)$ spectrum of water confined in a polymer membrane (Nafion). The elastic, quasi-elastic and inelastic zones of the spectrum are clearly visible. One can see that the reality of a measurement is far from the idyllic vision presented in a) where the spectral bands are fine and the excitations clearly defined. The complexity of the experimental spectrum is partly due to the sample dynamics but also to the intrinsic energy resolution of the spectrometer (gray curve, see also Fig.13). c) The considerations exposed in Fig.13 showed that the energy resolution is Gaussian in the (Q, $\omega)$ domain. Fourier transform of a Gaussian (of HWHM $\Delta \omega$ ) and a Lorentzian (of HWHM $\Gamma$ ) in the energy domain are, in the time domain, a Gaussian (HWHM: $1 / \Delta \omega$ ) and an exponential decay (with correlation time $\tau=\hbar / \Gamma$ ), respectively. The point to keep in mind is that in both cases, the broader the curve in energy, the narrower the curve in time. Thus, the Fourier transform of the quasi-elastic line, wider than the energy resolution (curve b), is a temporal signal (purple exponential) narrower than the resolution (gray dotted curve in c). We can see on c) that to measure long times, the limiting factor is the maximum time corresponding to the resolution. Graphically, this translates into the fact that it is impossible to measure correlation time corresponding to exponentials located in the region above the gray dotted line. This area is "forbidden" as symbolizes the light gray area. The only way to measure longer correlation times is to increase the resolution in energy: the narrower the Gaussian elastic peak (energy domain) the broader its Fourier transform (time domain). However, the price to pay to increase the resolution is usually a decrease in flux.

\footnotetext{
${ }^{13}$ A decaying exponential with a correlation time of $10 \mathrm{ps}$ is, in the energy domain, a Lorentzian line with HWHM $\Gamma=0.658 / 10=65.8 \mu \mathrm{eV}$.
} 


\section{The tools at work: A multiscale study of a polymer melt in bulk and under nanometric confinement. Connection to NMR}

After decades of developments, very high-achieving theoretical tools are now on hand to finely interpret both the structural and dynamical behaviors of polymeric systems. The theoretical models describing the physics of bulk polymer melts have, for example, made possible to establish a connection between the rheological properties and the dynamics of the chains at the microscopic scale. For long entangling chains, the diameter of the reptation tube, $d_{R e p}$, controls the level of the plateau of the stress relaxation modulus $G_{e} \approx 1 / d_{\text {Rep }}^{2}$ and, in turn, the polymer viscosity. Polymer physics is therefore a canonic example of a successful multiscale approach of a complex system in bulk. Nevertheless, despite the key practical and industrial relevance of such situations, the peculiar properties of polymers in interfacial situations or in strong confinement are far from being fully understood.

In the present section, we address the different dynamical modes of a high-mass entangled polymer chain: the local monomer dynamics, the Rouse modes and the reptation process. This study exemplifies that, used in conjunction with hydrogen/deuterium isotopic effects, high resolution QENS can be bridged to the Zero Average Contrast (ZAC) method to probe, in a non destructive way, the dynamics of a single polymer chain in bulk but also under severe nanometric confinement. Connection and complementarity of the neutron derived analysis with Pulsed-Field Gradient and Relaxation NMR techniques are also discussed.

\subsection{Polymer dynamics in a nutshell}

A polymer chain experiences different dynamical regimes [16, 17], spread over broad time and length scales. At short time and at the local scale ( $1 \mathrm{~ns}, 50 \AA$ ), the chain just experiences random entropic forces and freely fluctuates in an isotropic way. This is the Rouse regime. At longer times and larger scale (100 ns, $500 \AA$ ), the neighboring chains induce a topological constraint and the chain fluctuates alongside a fictive tube [18]: the so-called reptation tube (regime of the local reptation). The tube diameter is similar to the distance between two entanglements i.e. around $50 \AA$. Then, at even longer times $(1000 \mathrm{~ns})$, in the so-called full reptation regime, the chain can finally escape the tube. Such a microscopic model is extremely useful and efficient, since with very few parameters (a monomeric friction coefficient and the length of a chain segment), it is possible to bridge the local dynamics to macroscopic properties like, for example, the polymer viscosity.

In numerous scientific fields, reducing the dimensions of a physical system down to a scale that matches the characteristic sizes of its natural fluctuations in bulk, usually leads to unexpected and surprising new physical behaviors [19]. In soft-matter, despite the key practical and industrial relevance of such situations, the peculiar properties of polymers in interfacial situations [20] or strong confinement [21] are far from being fully understood. Several theoretical approaches have addressed the issue of the polymer conformation, when a melt is confined inside cylindrical pores [22]. It has been proposed that, under confinement, a modification of the monomer-monomer excluded volume interaction could lead to an increase of the longest polymer relaxation time and therefore of the confined polymer viscosity [23].

As a matter of fact, even in the recent past, confinement was achieved in media with no macroscopic orientation: "powders" in the crystallographic sense of this word. This has two consequences when probing the physical properties of confined fluids:

- first, all the retrieved structural and dynamical information are blurred by isotropic average: key points like the relative directions of the motions of a molecule with respect to the pore (radial or longitudinal) are basically lost. 
a)

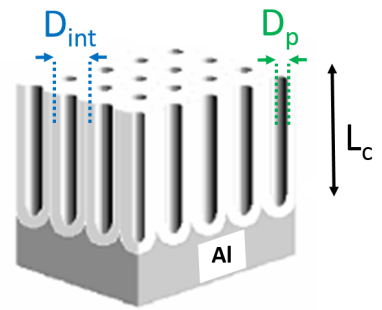

b)

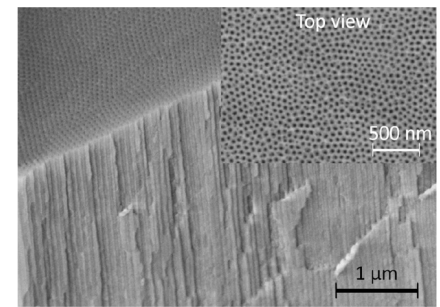

Figure 16. a) Schematic structure of an anodized alumina membrane (AAO). From [24]. The membrane topology can be described by the pore diameter, $D_{p}$, the inter-pores distance, $D_{i n t}$, and the channels length, $L_{c}$. b) Scanning Electron Microscope image of a typical AAO membrane. The highly oriented array of cylindrical pores (showing an imperfect hexagonal arrangement, see inset) goes from the top down to the bulk of the membrane. We illustrate in section 4.3 how the macroscopic orientation of such a pore network can be used to lift the limitations of the powder average. From [25].

- at a second level, the tortuosity (imposed by the pore morphology and/or the random spatial arrangement of the powder grains) is making it extremely challenging to link the local transport properties (few $\AA$ or nm), like the self-diffusion coefficient, to the macroscopic transport flow of the material.

Following recent progresses in inorganic chemistry, porous media with pores showing macroscopic orientation are now available (Fig.16). The "powder average" limitation can therefore be easily overcome by simply orienting the porous sample in a measuring device (confocal microscope, NMR, infra-red, neutron spectrometers ... ). It is then relatively easy to firmly disentangle dynamical phenomena in the parallel or radial direction of the pore axis.

The Rouse model successfully describes the physics of short-chain polymer melts [26]. This model ignores the atomic structure, to assimilate the polymer to an ideal (or Gaussian) chain made by a linear succession of beads (with a diameter $\sigma$, called the Kuhn length ${ }^{14}$ ) interacting through a harmonic potential. The conformational entropy is the restoring force that prevents large excursions from the average equilibrium conformation. The full mathematical development of the model leads to the following intermediate scattering function:

$$
I^{\text {Rouse }}(Q, t)=e^{-\frac{2}{\sqrt{\pi}}\left(\frac{3 k_{B} T}{\sigma^{2} \zeta_{0}} Q^{4} t\right)^{\frac{1}{2}}}
$$

where $k_{B}$ is the Boltzmann constant, $T$ the temperature, $\zeta_{0}$ the monomeric friction coefficient and $\sigma$ the length of a chain segment. $W=3 \cdot k_{B} T \cdot \zeta_{0}{ }^{-1} \sigma^{-2}$, the elemental Rouse rate, is the quantity generally used to characterize the correlation time associated to the polymer dynamics. This expression also writes:

$$
I^{\text {Rouse }}(Q, t)=e^{-\left(\frac{t}{\tau}\right)^{\beta}}
$$

One now clearly sees that Eq.41 is a stretched exponential, with the stretched exponent $\beta$ exactly equal to $1 / 2$. Also, within the framework of the Rouse model, the system is characterized by a single correlation time $\tau$ and $\tau$ follows a $Q^{-4}$ power law. The Rouse model is able to describe the polymer

\footnotetext{
${ }^{14} \mathrm{~A}$ real polymer chain can be described as an equivalent so-called Kuhn chain made of a succession of $N$ connected Kuhn segments (or beads) of Kuhn length $\sigma$. This theoretical treatment introduced by Kuhn, makes it possible to assimilate a real complex polymer chain to a random coil i.e. a random walk of the Kuhn segments [17].
} 
dynamics up to $Q$ values around $0.3 \AA^{-1}$ with a remarkable accuracy. At higher $Q$, the detailed local structure and dynamics of the chain under scrutiny give strong contribution and Eq.41 fails to account for the data. Despite significant deviations from the ideal form of the Rouse model and therefore a lack of clear physical significance, stretched exponentials are often used to account for QENS and NMR relaxation data from polymer melts. In many cases, $\beta$ is found to differ from its ideal 0.5 value and becomes $Q$ dependent, $\tau$ does not follow a power law in $Q^{-4}$ and these deviations change from polymer to polymer.

\subsection{Local polymer dynamics: A neutron-derived physical model}

\subsubsection{QENS derived dynamical susceptibility as a tool to determine the actual number of dynamical contributions}

This section is devoted to the definition of a model to account for the dynamics of bulk PEO as sensed on the typical structural and dynamical ranges of a ToF experiment (few $\AA$ and few tens of ps), i.e. in the situation where the pure stretched exponential description of a polymer dynamics fails.

As this has been shown in section 2.5.3, mean-square displacements as measured by incoherent neutron scattering (elastic scans) can be informative and provide a global view of the dynamics of a system. But we need to clearly state that, when the instrumental resolution (in energy) of the spectrometer matches the relaxation characteristic times of the system under investigation, the way to take the best of a QENS experiment, is to undertake a full measurement of the inelastic and/or quasi-elastic signal. Then, fitting the experimental data with a meaningful model can lead to more detailed insights in the physics of the system.

To illustrate this strategy, we start with an elastic-scan measurement (Fig.8) to obtain a global view of the dynamics of the confined p olymer. Then, we attempt to derive a more physically meaningful model of the local $(0.1 \mathrm{~nm})$, short time $(10 \mathrm{ps})$ dynamics of a linear polymer like PEO. We consider $i)$ orientational diffusion along the polymer chain, ii) local conformational transitions and iii) long-time, large-scale motions. The model takes into account the spatial component of the local dynamics, described in terms of the scattering vector $Q$.

PEO can be confined within the porous structure of an AAO membrane by directly melting the polymer under vacuum onto the membrane. The filling process is completed after a few $\mathrm{d}$ ays. Under confinement the melting o ccurs a t a s ignificantly lo wer te mperature $\left(T_{M}=317 \mathrm{~K}\right)$ th an in bulk $\left(T_{\text {MBulk }}=333 \mathrm{~K}\right)$ : a clear Gibbs-Thomson effect is detected. This strong thermodynamical effect has also a signature, in terms of local mobility, on the polymer mean-square displacement measured by incoherent neutron scattering (Fig.8).

In order to unveil the various dynamical processes involved, full QENS measurements are shown in Fig. 17 in terms of the dynamical susceptibility function:

$$
\chi^{\prime \prime}(Q, \omega)=S(Q, \omega) / n_{B}(\omega, T)
$$

where $n_{B}(\omega, T)=\left(1-\exp \left(-\frac{\hbar \omega}{k_{B} T}\right)\right)^{-1}$ is the Bose population factor, $T$ the temperature in Kelvin, $k_{B}$ the Boltzmann constant and $\hbar$ the Planck constant divided by $2 \pi$. In quasi-elastic scattering, a relaxation mechanism with characteristic time $\tau$ appears, in $S(Q, \omega)$, as a quasi-elastic broadening of HWHM $\Gamma=\frac{\hbar}{\tau}$ and, in $\chi^{\prime \prime}(Q, \omega)$, as a band centered at $\omega \approx \Gamma$. Two dynamical regions can be clearly identified in the bulk polymer (Fig.17a). A low-energy dispersive band $\left(E \approx 0.07 \mathrm{meV}\right.$ at $\left.Q_{0}=0.55 \AA^{-1}\right)$ merges at higher $Q$ with a non-dispersive band $(E \approx 5 \mathrm{meV})$, giving rise to a single broad band for $Q$ values above $1.7 \AA^{-1}$. This purely qualitative analysis leads to the conclusion that the dynamics of the systems can be accounted for by a system with at least two distinct correlations times: one being 

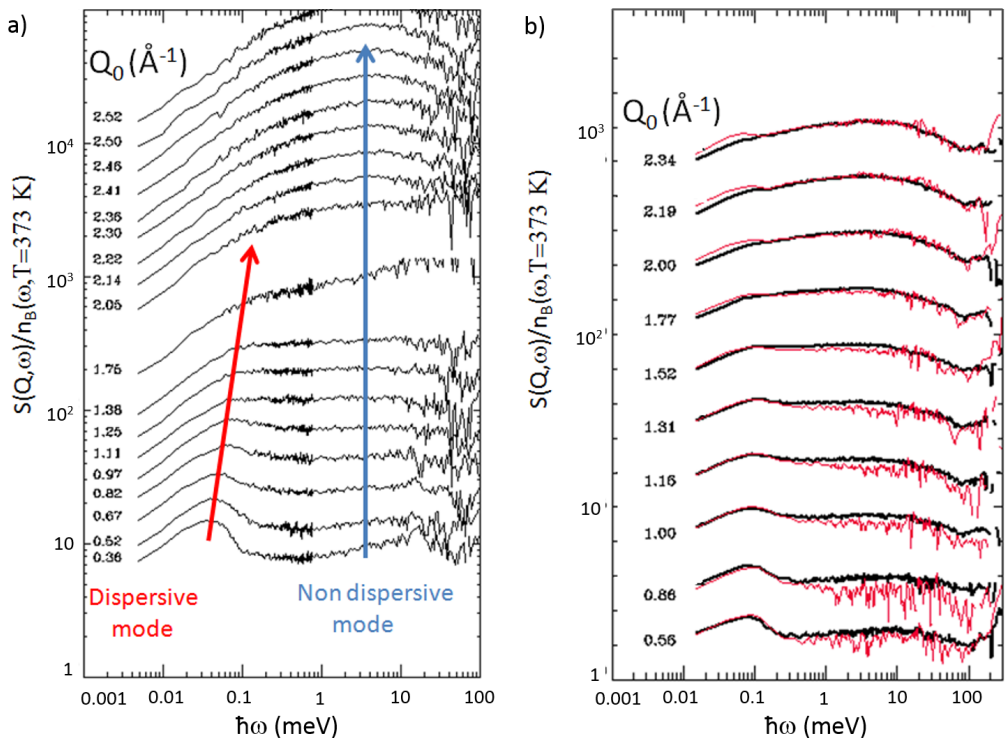

Figure 17. Q dependence of the dynamical susceptibility spectra, $\chi$ " $(Q, \omega) v s \hbar \omega$, calculated from the QENS data of: a) bulk PEO (100 kg/mol, QENS spectrometer at ANL/IPNS) and b) PEO confined in AAO with 35 $\mathrm{nm}$ diameter pores (red line, Mibémol spectrometer at LLB). Curves are shifted for clarity and values of $Q_{0}$, the elastic scattering vector is indicated. For the confined sample, the corresponding signal of bulk PEO is shown (black line). In all cases, $\mathrm{T}=373 \mathrm{~K}$. From [27].

dispersive, the other being $Q$ independent. The same conclusion applies for the confined polymer (Fig.17b).

\subsubsection{A physical appealing model of the local polymer dynamics: The building blocks}

\section{- Local conformational transitions.}

If the conformational motions experienced by a monomer are characterized by a single correlation time $\tau_{0}$, the corresponding scattering function is:

$$
S_{i n c}^{\text {Conf }}(Q, \omega)=A_{0}(Q) \cdot \delta(\omega)+\frac{1-A_{0}(Q)}{\pi} \cdot L_{\text {inc }}^{\text {Conf }}(Q, \omega)
$$

where $L_{\text {inc }}^{\text {Conf }}(Q, \omega)$ is a Lorentzian function of HWHM $\hbar / \tau_{0}(Q)$ and $A_{0}(Q)$ is the Elastic Incoherent Structure Factor (EISF) associated with the geometry of the motion. For a purely incoherent sample, as the one we are dealing with here, the EISF is the infinite time limit of the intermediate scattering function $I_{\text {Self }}(Q, t)$. The $Q$ dependence of $I_{S e l f}(Q, t)$ at $t=+\infty$, is the form factor of the localization volume of the particle (see section 2.5.2 and Fig.7).

-Local PEO dynamics: A mechanism unveiled by $2 D{ }^{13} C$ solid-state NMR. Extrapolation to $S(Q, \omega)$.

In the case of crystalline PEO, few $2 D{ }^{13} C$ solid-state NMR experiments have shown [28] that the local chain conformational transitions (few $\AA$ ) can be described as an helical jump (HJ) motion. This process has been detected at $225 \mathrm{~K}$, i.e. just few kelvins above the glass transition and is therefore 
a)

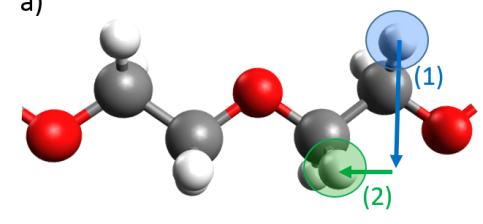

b)

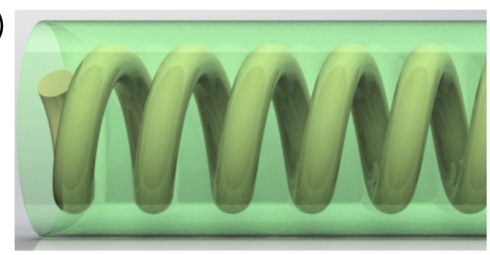

Figure 18. a) Schematic representation of a PEO chain $\left(-\left[\mathrm{CH}_{2}-\mathrm{CH}_{2}-\mathrm{O}-\right]_{n}\right)$ with the two fundamental motions involved described in this section. A local "jump over two sites" conformational change (noted 1) with non dispersive correlation time $\tau_{0}$ and EISF $A_{0}(Q)$, is coupled to a translation of the whole polymer chain (noted 2) with a dispersive (in $Q^{-2}$ ) correlation time $\tau_{1}$ and EISF $A_{1}(Q)$. Altogether, those two motions give rise to a helical jump $(\mathrm{HJ})$ motion. Its envelope is represented in green on sketch b). The jump distance derived from $A_{0}(Q)$ is about $2 \AA$. This HJ motion extends up to $26 \AA . A_{1}(Q)$ has therefore a form factor of a cylinder of $2 \AA$ in radius and $26 \AA$ in length. As a summary, we reach the conclusion that in bulk the whole PEO polymer chain is sliding, as a whole, along its own physical contour.

very slow. It involves a rotation of a monomer by $180^{\circ}$ with a concomitant chain translation of one monomer unit (Fig.18).

This translation can be described as a Random Walk in Continuous Time (RWCT), a generalization of the discrete random walk in which the waiting time probability density shows a time dependence $\Psi(t)$, instead of being a Dirac function corresponding to a single residence time (like in jump diffusion, see section 2.5.1). If $\Psi(t)$ is Poissonian, which is the case for conformational changes in PEO melt [29], the autocorrelation function of the motion is given by:

$$
G^{H J}(t)=e^{-t / \tau_{1}} I_{0}\left(t / \tau_{1}\right)
$$

where $\tau_{1}$ is the rate of the conformational change and $I_{0}$ is the zero-order modified Bessel function of the first kind. The long time-scales over which the HJ motions take place at $225 \mathrm{~K}$ are inaccessible by QENS. However, we can expect these motions to be still present above the melting point, but several orders of magnitudes faster, and thus within the dynamical range of QENS. Due to the effect of damping, originating from both the averaged dynamics of the neighboring chains and the presence of entanglements, one can expect that the diffusion of the polymer chains involved in the HJ motions should not extend beyond a few nanometers. These diffusive motions being localized, they give rise to a scattering function of the form:

$$
S_{\text {inc }}^{\text {Diff }}(Q, \omega)=A_{1}(Q) \cdot \delta(\omega)+\frac{1-A_{1}(Q)}{\pi} S_{\text {inc }}^{H J}(Q, \omega)
$$

where $A_{1}(Q)$ is the form factor (EISF) of the volume over which the diffusion takes place and $S_{\text {inc }}^{H J}(Q, \omega)$ is the Fourier transform of Eq.45 with $\tau_{1}$ being $Q$-dependent.

\section{- Long time and large scale rheology-related motions.}

At times beyond those where this atomic description applies, larger scale viscoelastic properties have to be taken into account. The present work focuses on high $Q\left(>0.3 \AA^{-1}\right)$ data in which these larger scale motions are not likely to be accurately described. For the present purposes, they will be accounted for by a single relaxation time $\tau_{2}(Q)$, leading to the scattering function:

$$
S_{\text {inc }}^{\text {Glob }}(Q, \omega)=\frac{1}{\pi} \int e^{-t / \tau_{2}} \cdot e^{-i(Q r-\omega t)} \cdot d t
$$


Assuming that the three different dynamical mechanisms described above are uncorrelated, the final scattering function $S_{i n c}^{P E O}(Q, \omega)$, describing in full the PEO dynamics, is a convolution of EQ.44, 46 and 47. The correlation time $\tau_{0}$, representing the local reorientational dynamics, is expected to be much shorter than $\tau_{1}$ and $\tau_{2}$ so that, to a good approximation:

$$
\begin{gathered}
S_{\text {inc }}^{\text {Conf }}(Q, \omega) \otimes S_{i n c}^{H J}(Q, \omega) \approx S_{\text {inc }}^{\text {Conf }}(Q, \omega) \\
S_{i n c}^{C o n f}(Q, \omega) \otimes S_{i n c}^{G l o b}(Q, \omega) \approx S_{\text {inc }}^{\text {Conf }}(Q, \omega)
\end{gathered}
$$

and $S_{\text {inc }}^{P E O}(Q, \omega)$ simplifies [27] to:

$$
S_{i n c}^{P E O}(Q, \omega)=B_{0}(Q) \cdot \delta(\omega)+B_{1}(Q) \cdot S_{i n c}^{G l o b}(Q, \omega) \otimes S_{i n c}^{H J}(Q, \omega)+B_{2}(Q) \cdot S_{i n c}^{C o n f}(Q, \omega)
$$

where $B_{0}(Q), B_{1}(Q)$ and $B_{2}(Q)$ are respectively the intensities of the elastic peak, a narrow and a broad quasi-elastic contribution. It is important to note that by fitting the QENS data with Eq.50, we impose the time (or equivalently the energy) dependence of the quasi-elastic signal (i.e. the shape of the contributions), but their relative intensities are free parameters. For each $Q$ value, from $B_{0}(Q)$, $B_{1}(Q)$ and $B_{2}(Q)$ we can derive $A_{0}(Q)$ and $A_{1}(Q)$, the form factors of the conformational changes and of the extension of the HJ motion (Fig.19).
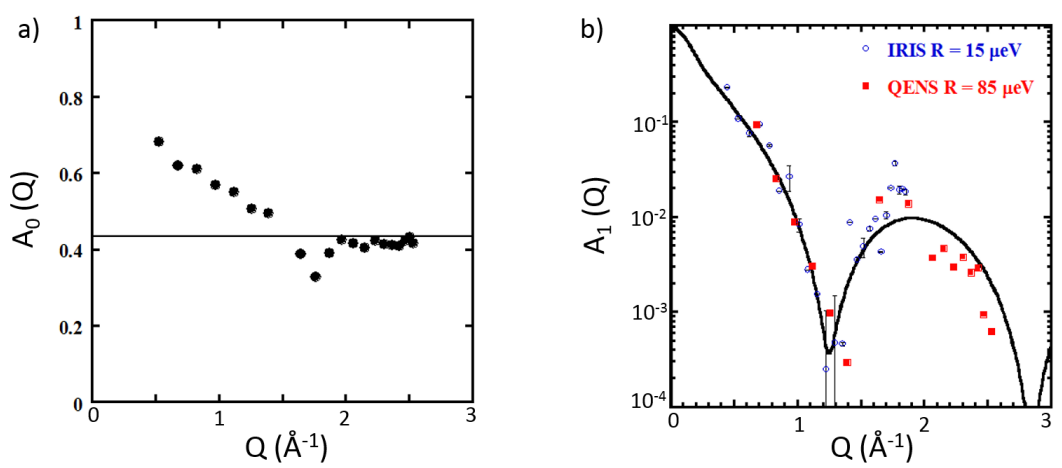

Figure 19. a) $A_{0}(Q)$, form factor (EISF) for the conformational changes, derived from the $B_{0}(Q), B_{1}(Q)$ and $B_{2}(Q)$ intensities of Eq.50. The full horizontal line indicates the level $(1-a)$ of the weighting factor balancing the short and long correlation time contributions to the ${ }^{13} \mathrm{C}$ spin-lattice relaxation NMR spectral density (see Eq.51). b): Semi-log plot of $A_{1}(Q)$ deduced from the fit of IRIS data (RAL/ISIS, UK, high resolution, low-Q, in blue) and QENS data (ANL/IPNS, USA, low-resolution, high-Q data, in red), with Eq.50. The full line represents the form factor of a cylinder of $2 \AA$ radius and $26 \AA$ long. From [27].

We find the $Q$ dependencies of the three correlation times of the model $\tau_{0}, \tau_{1}$ and $\tau_{2}$, to be respectively consistent with $Q^{0}, Q^{-2}$ and $Q^{-4}$ power laws (Fig.20). At $373 \mathrm{~K}$, the polymer dynamics is described in terms of transverse motions of the chain segments over a distance of few $\mathrm{nm}$, with a local monomeric diffusion coefficient derived from the $Q^{-2}$ dependence of $\tau_{1}: D_{\text {Mono }}=1.78 \times 10^{-9} \mathrm{~m}^{2} . \mathrm{s}^{-1}$. From this value, a monomeric friction coefficient $\zeta_{0}=k_{B} T / D_{\text {Mono }}=2.89 \times 10^{-12}$ N.s. I $^{-1}$ can be derived and used as numerical input to the Doi-Edwards theory [16]. This leads [27] to a chain centerof-mass diffusion coefficient $D_{c m}=9.4 \times 10^{-15} \mathrm{~m}^{2} \cdot \mathrm{s}^{-1}$. This value is in very good agreement with Pulsed Field Gradient NMR (PFG-NMR) data [30] (Fig.20).

Within the framework of this model, the two distinct dynamical modes clearly detected in the dynamical susceptibility plots of the bulk polymer (Fig.17) can be attributed to the helical jump motion 

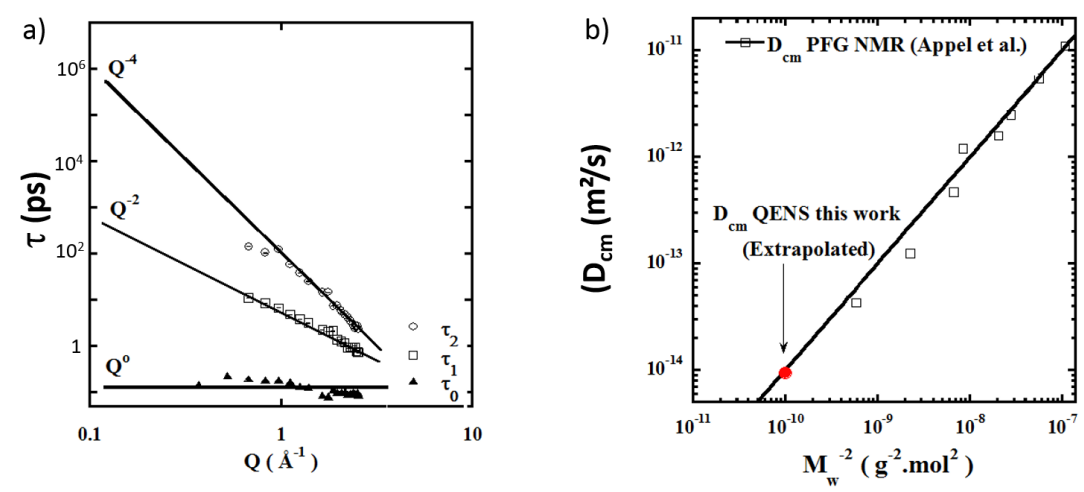

Figure 20. a) Correlation times $\tau_{0}, \tau_{1}$ and $\tau_{2}$ derived from the fits of the QENS data with Eq.50. The straight lines indicate the power-law behaviors. From the $Q^{-2}$ dependence of $\tau_{1}$, we derive a monomeric friction coefficient $\zeta_{0}=2.89 \times 10^{-12} N . s . m^{-1}$. From this value, we can derive the polymer chain center-of-mass diffusion coefficient: $D_{c m}=k_{B} \cdot T \cdot M_{e} /\left(3 \cdot \zeta_{0} \cdot M_{W}\right)$ with $\left.M_{W}=100 \mathrm{~kg} / \mathrm{mol} . \mathrm{b}\right)$ Molecular weight dependence of the PEO chain centerof-mass self-diffusion coefficient $D_{c m}$ (PFG-NMR data from [30]). The $D_{c m}$ value derived from the neutron data in a), is shown as a red point. From [27].

( $Q^{-2}$ dependent correlation time $\tau_{1}$, narrow dispersive band) and to the non dispersive conformational jump with correlation time $\tau_{0}$ (broad dispersive band around $5 \mathrm{meV}$ ). Since no significant difference is detected at $373 \mathrm{~K}$ in the susceptibility plot of the $\mathrm{PEO}$ confined in $35 \mathrm{~nm}$ pores, such an interpretation seems to hold on that case also.

The high $Q$ limit of $A_{0}(Q)$ around 0.5 is characteristic of a jump motion over two sites (i.e. the probability to be on one site is $1 / 2$ ). From the $Q$ dependence of $A_{0}(Q)$ we can estimate a jump distance of about $2 \AA$. The experimental values of $A_{1}(Q)$ are consistent with a form factor of a cylinder of $2 \AA$ in radius and $26 \AA$ in length. From this geometrical information and the $Q$ dependence of the correlation times of the model (Fig.20), we propose the microscopic mechanism of a PEO polymer chain dynamics shown in Fig.18: the whole PEO polymer chain is sliding as a whole along its own physical contour.

\subsubsection{Bridging the QENS and the NMR relaxation models}

Detailed molecular dynamics can be also assessed by NMR. Hall and Helfand (HH) [31] derived a bimodal autocorrelation function to describe damped conformational changes along a polymer chain. Subsequently, Dejean de la Batie et al. [32] showed that this autocorrelation function could not account for the high value of the spin-lattice relaxation time $T_{1}$ observed in ${ }^{13} \mathrm{C}$ spin-lattice relaxation time experiments. To explain their data, they complemented the $\mathrm{HH}$ model with an additional fast, small-amplitude anisotropic motion that they identified as librations of $\mathrm{C}-\mathrm{H}$ vectors. They propose the so-called Dejean-Laupretre-Monnerie (DLM) model:

$$
G(t)=(1-a) \cdot e^{-t / \tau_{1}} \cdot e^{-t / \tau_{2}} \cdot I_{0}\left(t / \tau_{1}\right)+a \cdot e^{-t / \tau_{0}}
$$

In the first term, following Hall and Helfand [31], $\tau_{1}$ is the correlation time associated with the conformational jumps responsible for orientational diffusion along the polymer chain, while $\tau_{2}$ represents a damping mechanism consisting of either non-propagating isolated motions or distortions of the chain with respect to its stable local conformation. The fast relaxation $\tau_{0}$ corresponds to the correlation time 
of librations of $\mathrm{C}-\mathrm{H}$ vectors inside a cone of half-angle $\theta$ and $a$ is a geometrical factor depending on $\cos (\theta)$.

In the case of PEO with $M_{w}=9200 \mathrm{~g} / \mathrm{mol}$ at $373 \mathrm{~K}$, Dejean de la Batie et al., [32] report $\tau_{1} \approx 4 \mathrm{ps,}$ $\tau_{1} / \tau_{0}=200, \tau_{1} / \tau_{2}=200$, and $\mathrm{a}=0.56$, corresponding to the libration of a C-H bond in a cone of half-angle $\theta=40^{\circ} \mathrm{C}$.

There is clearly a formal similarity between the DLM and the QENS model. For the sake of comparison, we express the latter in the variables $Q$ and $t$ :

$$
I(Q, t)=A_{0}(Q) \cdot A_{1}(Q)+A_{0}(Q)\left(1-A_{1}(Q)\right) \cdot e^{-t / \tau_{1}} \cdot e^{-t / \tau_{2}} \cdot I_{0}\left(t / \tau_{1}\right)+\left(1-A_{0}(Q)\right) \cdot e^{-t / \tau_{2}}
$$

As immediately apparent from a comparison of Eq.51 and 52, the presence of the variable $Q$ could lead to the impression that it is difficult to directly compare models derived from QENS and NMR data. Based on the simple qualitative argument that the dipole-dipole interactions giving rise to the NMR $T_{1}$ relaxation is short-ranged, we can argue that NMR assesses the atomic scale dynamics (two or three covalent bonds). This corresponds to a $Q$ range of $Q \approx 2 \pi / 3 \approx 2 \AA^{-1}$. Replacing $A_{0}(Q)$ and $A_{1}(Q)$ by 0.44 and 0 , their numerical values at $Q \approx 2 \AA^{-1}$ (Fig.19), Eq.52 exactly reduces to Eq.51. Therefore, starting from a $2 D{ }^{13} C$ solid-state derived molecular motion (HJ), we have proposed a detailed model of the PEO motion at the molecular level. The correlation times and characteristic distances of the model are respectively consistent with PFG-NMR and $T_{1}$ NMR relaxation data.

\subsection{The Rouse regime}

Neutron Spin-Echo (NSE) is in principle a suitable technique to match the time and spatial scales relevant to the Rouse regime. Moreover, by specific orientation of the macroscopically oriented AAO membranes with respect to the incident neutron beam (see Fig.21) of a NSE spectrometer, it is possible to probe the intermediate self-correlation function perpendicular (radial dynamics) and parallel (longitudinal dynamics) to the axis of the cylindrical AAO pores.
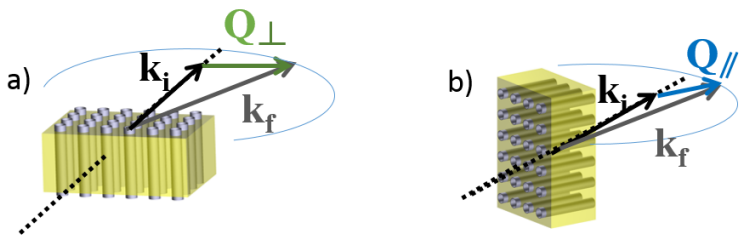

Figure 21. By orienting macroscopically oriented porous samples with respect to the neutron beam, the dynamics perpendicular/radial (a) or parallel (b) to the pores can be independently retrieved. From [33].

Severe restriction applies to NSE measurements: the dynamics is assessed through the polarization, $\langle P\rangle$, of the neutron beam after interaction with the sample. $\langle P\rangle$ is a function of $I_{c o h}$ and $I_{\text {inc }}$ the coherent and incoherent scattered intensities: $\langle P\rangle=I_{\text {coh }}-I_{\text {inc }} / 3$. Therefore, no observable are available (or with very poor statistics) in $Q$ ranges where $I_{c o h} \approx I_{\text {inc }} / 3$. The experimental polarization of bulk and AAO confined PEO is shown in Fig.22a. As this has been stated earlier, the Rouse model applies for $Q$ below $0.3 \AA^{-1}$. For $Q<0.3 \AA^{-1}$, the polarization is good for both samples, but a full $I(Q, t)$ measurement shows that the signal is purely static (coherent elastic scattering of the aluminum cell and/or the alumina matrix). In the $0.3 \AA^{-1}$ region, the polarization of the bulk makes the $I(Q, t)$ accessible, but the polarization of the confined sample is almost null. Altogether, the smallest $Q$ 
available where the sample dynamics is in the NSE time window and the polarization is not null for both the bulk and the confined polymer is $0.62 \AA^{-1}$. The corresponding data are shown on Fig.22b.
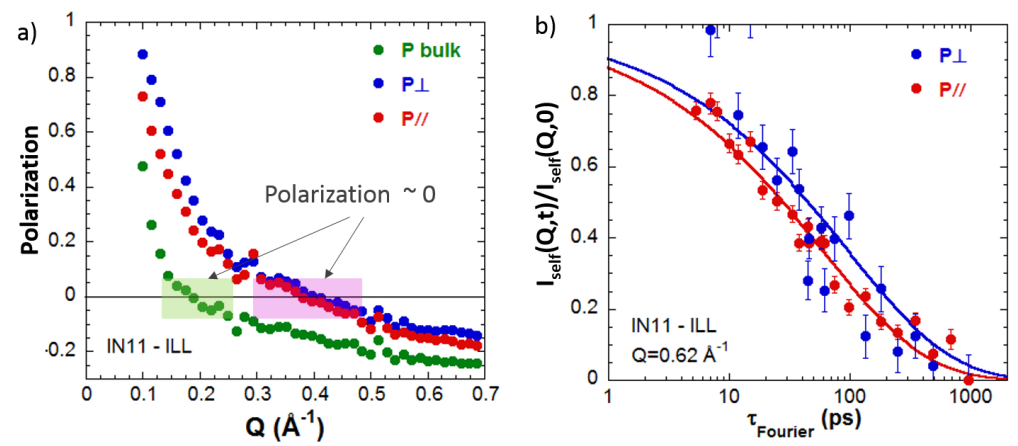

Figure 22. NSE results. The sample is ${ }^{h} \mathrm{PEO}\left(M_{w}=35000 \mathrm{~g} \cdot \mathrm{mol}^{-1}\right)$ confined within the macroscopically oriented structure of an AAO (24 nm in diameter), at $373 \mathrm{~K}$. AAO membranes being macroscopically oriented, specific orientation of the sample in the neutron beam makes it possible to access the dynamics parallel and perpendicular to the pores. a) Polarization of the neutron beam for bulk PEO, and for the two orientations of the AAO confined sample. b) Neutron spin-echo measurements (IN11-ILL) of $I_{S e l f}(Q, t)$ (Eq.42), the self intermediate scattering function, at $Q=0.62 \AA^{-1}$. No differences are detected between the polymer dynamics perpendicular (blue symbols) and parallel (red symbols) to the axis of the cylindrical AAO pores. From [34].

Even at this rather large $Q$ value the Rouse model Eq.41 holds (we do observe a master curve in Fig.23). W. $\sigma^{4}$ values of $11 \pm 2,3.3 \pm 0.7$, and $2.0 \pm 0.5 \AA^{4} \cdot p s^{-1}$ have been measured for the bulk PEO, the PEO confined in the parallel (alongside the AAO pore axis) and perpendicular direction, respectively. Within the statistics, at this rather local scale, no dynamical difference can be detected between the radial and longitudinal dynamics. However, under confinement the polymer dynamics decreases by a factor of about four.
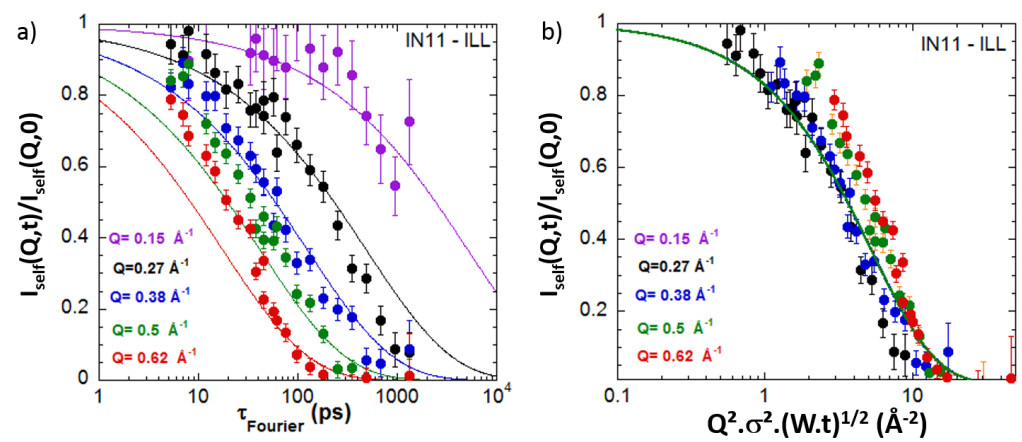

Figure 23. a) NSE spectra of bulk ${ }^{h} \operatorname{PEO}\left(M_{w}=35000 \mathrm{~g} \cdot \mathrm{mol}^{-1}\right)$ for various $Q$. b) Same data as in a) fitted with Eq.41. When plotted as a function of the Rouse variable $u=Q^{2} \sigma^{2} .(W \cdot t)^{1 / 2}$, the data fall on a master curve for $W \cdot \sigma^{4}$ values of $11 \pm 2 \AA^{4} \cdot p s^{-1}$. From [34]. 


\subsection{The reptation regime: Observing a single polymer chain (Zero Average Contrast)}

Since porous materials are usually very good scatterers, Small Angle Neutron Scattering (SANS) is a key technique for accessing their structure. But this advantage turns to a strong handicap when it comes to access the dynamics under confinement in the SANS regime: the fraction of intensity scattered inelastically by the confined material is several orders of magnitude smaller than the elastic contribution due to the static confining matrix. Then within the typical statistics of a neutron experiment (few \%) the dynamical information cannot be recovered. In the following section, we detail a general way to lift this difficulty.

As we have stated at the beginning of this section, the relevant time scale to probe polymer dynamics is at least one order of magnitude larger than the one assessed on the previous NSE experiment. A $Q$ range in the $10^{-2} \AA^{-1}$ region (now fully experimentally accessible on spin-echo spectrometers), is indeed much more appropriate. However, inelastic experiments in such a SANS region in situations of hard confinement are almost impossible: (i) the scattering of the confining porous matrix is usually extremely intense and (ii) since the porous material has no dynamics, this signal is purely elastic (Fig.24). As a consequence, the relevant information i.e. the inelastic scattering of the sole confined material is usually a negligible contribution to the total scattering, so that the related error bars prevent any practical use of the signal. The Zero Average Contrast (ZAC) technique makes it possible to overcome this difficulty (Fig.24).

The ZAC technique has been used to access the conformation of a polymer in semi-dilute solution, confined in the porous structure of Vycor [35]. A solution of hydrogenated (H) and deuterated (D) polymer chains in a solvent ( $\mathrm{S}$ ) have been confined in a porous medium (Vycor). If the solvent scattering length densities matches the scattering length densities of the confining matrix, the SANS intensity $I(Q)$, can be written as a sum of only three partial structure factors:

$$
I(Q)=\left(n_{D}-n_{0}\right)^{2} S_{D D}+\left(n_{H}-n_{0}\right)^{2} S_{H H}+2\left(n_{D}-n_{0}\right)\left(n_{H}-n_{0}\right) S_{H D}
$$

where, $n_{H}, n_{D}$ and $n_{0}$ are the neutron coherent scattering length densities of the hydrogenated polymer, the deuterated polymer and the solvent. In our case, the confining matrix can be assimilated to the solvent. If one makes the assumption that the $\mathrm{H}$ and $\mathrm{D}$ polymer chains are identical, Eq.53 becomes:

$$
I(Q)=\left(n_{D}-n_{H}\right)^{2} x_{d}\left(1-x_{d}\right) v \Phi N P(Q)+\left(x_{d} n_{D}+\left(1-x_{d}\right) n_{H}-n_{0}\right)^{2}\left[v \Phi N P(Q)+v \Phi^{2} Q(Q)\right]
$$

where $P(Q)$ and $Q(Q)$ are respectively the intra-chain and the inter-chain contributions, $x_{d}$ is the proportion of deuterated chains, $v$ the molecular volume of a monomer and $\Phi$ the polymer volume fraction. If $x_{d}$ can be set to meet the condition:

$$
x_{d} \cdot n_{D}+\left(1-x_{d}\right) \cdot n_{H}-n_{0}=0
$$

then, $I(Q)$ is only proportional to $P(Q)$, the form factor of a single chain. The coherent scattering length density of an AAO membrane can then be derived upon membrane impregnation by an isotopic mixture of water $\left(x_{d}=70 \mathrm{vol} \%\right): \rho_{A A O}=4.21 \times 10^{10} \mathrm{~cm}^{-2}$. The coherent scattering length density of $\mathrm{H}$ and D PEO being $\rho_{h P E O}=6.20 \times 10^{9} \mathrm{~cm}^{-2}$ and $\rho_{d P E O}=6.88 \times 10^{10} \mathrm{~cm}^{-2}$ respectively, the condition Eq.55 is fulfilled for $x_{d}=0.56$.

As shown in Fig.24a) by the perfect canceling out of the intense AAO peak, the H/D PEO mixture perfectly matches the AAO membrane. This is a direct experimental evidence that the polymer is fully and homogeneously confined within the AAO porous network. As shown by the strong similarity of the form factor, $P(Q)$, of a single confined polymer chain and bulk polymer chain form factor, no large scale significant structural change of the confined chain is detected by respect to its bulk analogue. In 

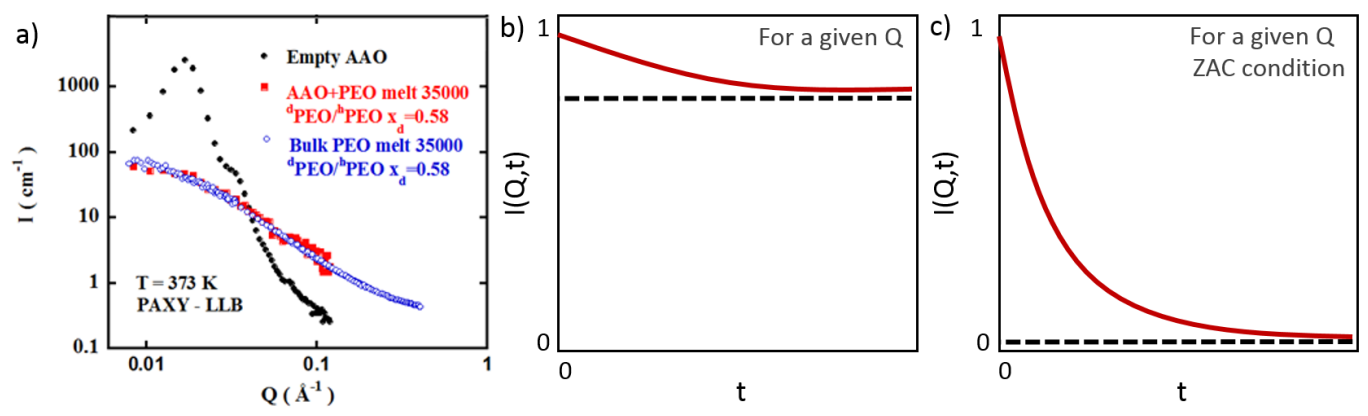

Figure 24. a) Relative intensities of an empty AAO (pore diameter $18 \mathrm{~nm}$ ) membrane and the same membrane filled with a ${ }^{d} P E O /{ }^{h} P E O$ mixture with a deuterated volume fraction $x_{d}=0.58$. The form factor of a single polymer chain measured on the same ${ }^{d} P E O /{ }^{h} P E O$ mixture in bulk (i.e. a typical bulk ZAC measurement) is shown for comparison. b) Due to the intense "parasitic" SANS elastic contribution (black dotted line) of the confining material, large scale NSE experiments to assess the dynamics of the confined material are, in practice, impossible (see text): the relevant inelastic contribution related to the confined polymer dynamics (full red line) has a negligible contribution. c) A way through is to combine ZAC conditions (to assess the signal of a single chain) with a matching condition of the confining material. Once matched, the SANS elastic intensity contribution of the membrane to the NSE $I(Q, t)$ signal becomes negligible (black dotted line), making the polymer correlation function (full red line) experimentally fully accessible (experimental data shown on Fig.25). Fig.a from [25].

particular, the radius of gyration $R_{G}=6.7 \pm 0.1 \mathrm{~nm}$ is not affected by the confinement.

As far as the dynamical aspect is concerned, the reptation mechanism in a tube with diameter $d_{R e p}$ leads to a transient localization of the chain. This is accounted for in $I_{\text {Chain }}(Q, t)$, the intermediate scattering function of a single polymer chain [26]:

$$
\frac{I_{\text {Chain }}(Q, t)}{I_{\text {Chain }}(Q, t=0)}=[1-p(Q)] \cdot F(Q, t)+p(Q)
$$

with $p(Q)=e^{-\frac{Q^{2} d_{R e p}^{2}}{36}}$, the form factor of the reptation tube. Note that $p(Q)$ shows a $Q$ dependent but time independent plateau. $\mathrm{F}(\mathrm{Q}, \mathrm{t})$ writes:

$$
F(Q, t)=e^{-\frac{u^{2}}{36}} \cdot \operatorname{erfc}\left(-\frac{u}{6}\right)
$$

where $u=Q^{2} \sqrt{W \sigma^{4} t}$ is the Rouse variable, $\sigma$ is the length of a chain segment and $W=3 \cdot k_{B} T \cdot \zeta_{0}^{-1} \sigma^{-2}$ is the elemental Rouse rate. $W$ is a function of $\zeta_{0}$, the monomeric friction coefficient, $k_{B}$ the Boltzmann constant and the temperature $\mathrm{T}$.

The NSE data of the bulk and confined polymer (Fig.25) follow the model Eq.56. A fit of the $I_{\text {Chain }}(Q, t)$ data set provides a very precise determination of both the temporal and structural parameters of Eq.56. The description of the confined PEO data with the fitted parameters $W \sigma^{4}=1778 \pm 63 \AA^{4} \mathrm{~ns}^{-1}$ and $d_{R e p}=67 \pm 3 \AA$ is very satisfactory (Fig.25). The same data treatment applied to the bulk signal leads to $W \sigma^{4}=2066 \pm 60 \AA^{4} \mathrm{~ns}^{-1}$ and $d_{R e p}=65 \pm 2 \AA$. Both the dynamics and the reptation diameter of the bulk and confined polymer are indistinguishable (Fig.25). This conclusion is supported by several recent independent results obtained by quasi-elastic neutron scattering (NSE included) [25, 36, 37].

We should nevertheless stress-out that quasi-elastic neutron scattering is sensitive to both translational 

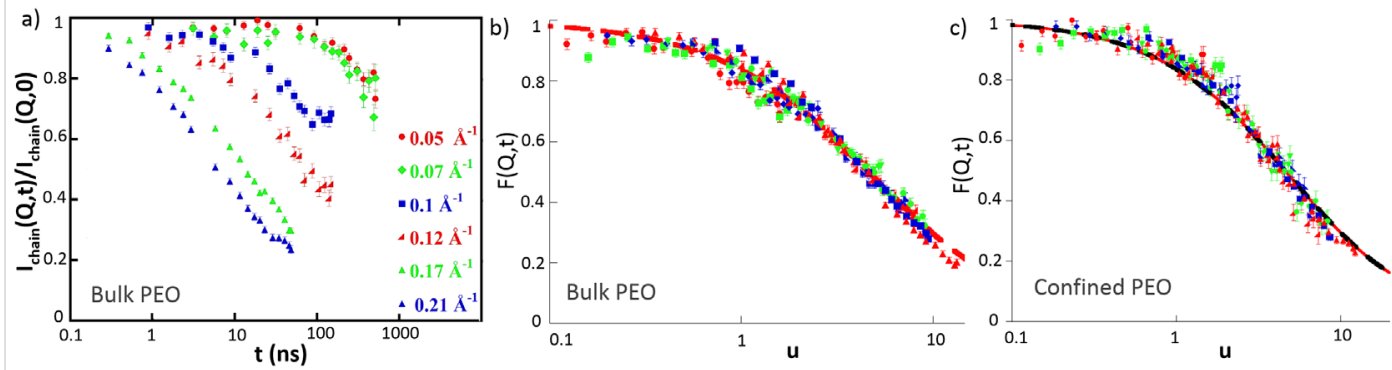

Figure 25. a) NSE intermediate scattering function of a single chain $35 \mathrm{~kg} \cdot \mathrm{mol}^{-1}$ PEO confined within an AAO membrane. The polymer is a mixture of deuterated and hydrogenated PEO $\left(x_{D}=56 \mathrm{vol} \%\right)$. This isotopic composition matching the AAO membrane, the intense elastic contribution due to the AAO membrane is canceled out and the scattered intensity is purely inelastic. $\left[I_{\text {Chain }}(Q, t) / I_{\text {Chain }}(Q, t=0)-p(Q)\right] /[(1-p(Q)]$ for the bulk (b) and confined polymer (c). The symbols used refer to the same $Q$ values than in a). The full and dotted lines stand for the master curve $F(Q, t)$ (Eq.56) with the fitted parameters $\left[W \sigma^{4}=1778 \pm 63 \AA^{4} \mathrm{~ns}^{-1} ; d_{R e p}=67 \pm 3\right.$ $\AA$ ], [ $\left.W \sigma^{4}=2066 \pm 60 \AA^{4} \mathrm{~ns}^{-1} ; d_{R e p}=65 \pm 2 \AA\right]$ for the confined and bulk polymer, respectively. From [25].

and rotational fluctuations. We observe in Fig.25, that the curvilinear Rouse motion of the local reptation regime (Doi-Edwards Regime II) satisfactorily describes the experimental $F(Q, t)$ of both the bulk and confined samples. Within the statistics of our NSE data, there is therefore no need to invoke any rotational contribution to describe the coherent intermediate scattering function of a single polymer chain.

\subsection{Multiscale study of a polymer melt: Conclusion}

Starting from a $2 D^{13} C$ solid-state NMR derived molecular motion (HJ), we have proposed a detailed model of the PEO motion at the molecular level. The correlation times and characteristic distances of the model are respectively consistent with PFG-NMR and $T_{1}$ NMR relaxation data. This physically meaningful model of the local $(\AA)$, short time (0.1-10 ps) dynamics of highly entangled ${ }^{h}$ PEO melt takes into account $i$ ) a helical jump motion along the polymer chain, ii) a local conformational transitions and iii) a long-time, large-scale motions. The model also accounts for the spatial dependence and geometry of these local motions via the Elastic Incoherent Structure Factors (EISF). To be able to detect the dynamics of the polymer chain confined in a nanometric cylindrical pore of an AAO membrane, we have combined SANS, contrast matching and ZAC with neutron spin-echo. This strategy provides a direct measurement of the time and $Q$ dependence of a single polymer chain (statistically averaged over all the chains of the sample). By interpreting the data within the framework of the reptation theory, we show that, in the conditions observed here (AAO pore radius $R_{P}=90 \pm 10 \AA$ and the radius of gyration of the $35 \mathrm{~kg} / \mathrm{mol}$ PEO $R_{g}=69 \pm 1 \AA$ ), confinement does not affect the dynamical property of the polymer chains nor the diameter of the reptation tube.

\section{Conclusion}

Inelastic and quasi-elastic neutron scattering scattering are central techniques to experimentally probe the dynamics of macromolecules (polymers or proteins). The dynamical information is measured in terms of the time or energy dependence of correlation functions of the atomic positions. The accessible time scale spans over decades from few fractions of picoseconds (time-of-flight techniques) to a 
few microseconds (Neutron spin-echo experiments). A specificity of the inelastic neutron techniques, is to directly and simultaneously probe the time and space correlation functions, so that the $Q$ dependence of the system characteristic time(s) can be measured. The dynamics of the system can then be understood by challenging the experimental observables with physical models accounting for spatial and temporal quantities. Altogether, the results presented here have illustrated the capabilities of neutron scattering in achieving a real multiscale approach ( $1 \mathrm{ps}$ to $0.6 \mu$ s and 0.1 to $40 \mathrm{~nm}$ ) of a canonical soft-matter physical system: a polymer melt.

We have highlighted how it is possible to take advantage of strong isotopic effects (for example hydrogen/deuterium) to disentangle specific dynamical contributions of complex architectures. We have in particular shown how, in conjunction with H/D isotopic effects, high resolution QENS/NSE can be bridged to the Zero Average Contrast (ZAC) method to probe, in a non destructive way, the dynamics (hence potentially the viscosity) of a single polymer chain in bulk but also under severe nanometric confinement. This is a situation where more classical experimental approaches, for example rheology, would have been impossible.

Last, it should be noted that the formalisms of inelastic neutron scattering and NMR are very similar. We have shown in several instances that firm connections between QENS and NMR techniques can be drawn and help to apprehend more finely the physics of a polymer melt. We hope that these few tentative connections will encourage new comers to the field to extend the numerous NMR/QENS complementarities to different soft-matter related systems.

\section{References}

[1] T. Perring, Lecture at the Oxford Neutron School (2007)

[2] G.L. Squires, Introduction to the Theory of Thermal Neutron Scattering (Courier Corporation, 1978)

[3] L. Van Hove, Physical Review 95, 249 (1954)

[4] F. Leclercq-Hugeux, M.V. Coulet, J.P. Gaspard, S. Pouget, J.M. Zanotti, Comptes Rendus Physique 8, 884 (2007)

[5] V.F. Sears, Neutron News 3, 26 (1992)

[6] J.M. Zanotti, Journal de Physique IV (Proceedings) 130, 87 (2005)

[7] M. Bée, Quasielastic Neutron Scattering (Adam Hilger, 1988)

[8] F. Volino, A.J. Dianoux, Molecular Physics 41, 271 (1980)

[9] V.F. Sears, Can. J. Phys. 44, 1279 (1966)

[10] J.M. Zanotti, K. Lagrené, N. Malikova, P. Judeinstein, K. Panesar, J. Ollivier, S. Rols, M. MayneL'Hermite, M. Pinault, P. Boulanger, Eur. Phys. J. Spec. Top. 213, 129 (2012)

[11] D. Klug, E. Whalley, E. Svensson, J. Root, V. Sears, Phys. Rev. B 44, 841 (1991)

[12] G.P. Johari, O. Andersson, Phys. Rev. B 73, 094202 (2006)

[13] M. Bastos, N. Alves, S. Maia, P. Gomes, A. Inaba, Y. Miyazaki, J.M. Zanotti, Physical Chemistry Chemical Physics 15, 16693 (2013)

[14] M.C. Rheinstädter, C. Ollinger, G. Fragneto, F. Demmel, T. Salditt, Phys. Rev. Lett. 93, 108107 (2004)

[15] J. Ollivier, J.M. Zanotti, Diffusion inélastique de neutrons par temps de vol (EDP Sciences, 2010), pp. 379-423

[16] M. Doi, S.F. Edwards, The Theory of Polymer Dynamics (Clarendon Press, 1988)

[17] M. Rubinstein, R.H. Colby, Polymer Physics (OUP Oxford, 2003)

[18] P.G. de Gennes, The Journal of Chemical Physics 55, 572 (1971) 
[19] R.L. Jones, S.K. Kumar, D.L. Ho, R.M. Briber, T.P. Russell, Nature 400, 22080 (1999)

[20] H. Bodiguel, C. Fretigny, Phys. Rev. Lett. 97, 266105 (2006)

[21] S. Sen, Y. Xie, S.K. Kumar, H. Yang, A. Bansal, D.L. Ho, L. Hall, J.B. Hooper, K.S. Schweizer, Physical review letters 98 (2007)

[22] F. Brochard, P.G. de Gennes, Journal de Physique Lettres pp. 399-401 (1979)

[23] A.N. Semenov, Phys. Rev. Lett. 80, 1908 (1998)

[24] F. Li, L. Zhang, R.M. Metzger, Chem. Mater. 10, 2470 (1998)

[25] K. Lagrené, J.M. Zanotti, M. Daoud, B. Farago, P. Judeinstein, Physical Review E 81 (2010)

[26] D. Richter, M. Monkenbusch, A. Arbe, J. Colmenero, Neutron Spin Echo in Polymer Systems (Springer, 2005)

[27] J.M. Zanotti, L.J. Smith, D.L. Price, M.L. Saboungi, Journal of Physics: Condensed Matter 18, S2391 (2006)

[28] K. Schmidt-Rohr, H.W. Spiess, Multidimensional Solid-state NMR and Polymers (Academic Press, 1994)

[29] O. Borodin, G.D. Smith, Macromolecules 33, 2273 (2000)

[30] M. Appel, G. Fleischer, Macromolecules 26, 5520 (1993)

[31] C.K. Hall, E. Helfand, The Journal of Chemical Physics 77, 3275 (1982)

[32] R. Dejean de La Batie, F. Lauprêtre, L. Monnerie, Macromolecules 21, 2045 (1988)

[33] Q. Berrod, F. Ferdeghini, P. Judeinstein, N. Genevaz, R. Ramos, A. Fournier, J. Dijon, J. Ollivier, S. Rols, D. Yu et al., Nanoscale 8, 7845 (2016)

[34] K. Lagrené, Ph.D. thesis, Paris Sud (2008), https://tel.archives-ouvertes.fr/ tel-00622540

[35] J. Lal, S. Sinha, L. Auvray, Journal de Physique II pp. 1597 - 1615 (1997)

[36] M. Krutyeva, J. Martin, A. Arbe, J. Colmenero, C. Mijangos, G.J. Schneider, T. Unruh, Y. Su, D. Richter, The Journal of Chemical Physics 131, 174901 (2009)

[37] J. Martín, M. Krutyeva, M. Monkenbusch, A. Arbe, J. Allgaier, A. Radulescu, P. Falus, J. Maiz, C. Mijangos, J. Colmenero et al., Phys. Rev. Lett. 104, 197801 (2010) 